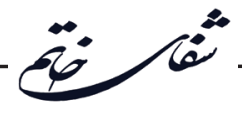

\section{Structural Model of Quality of Life in Patients with Breast Cancer: The Role of Psychological Hardiness, Religious Orientation, Perceived Social Protection and Self-Efficacy with Mediation of Resilience and Death Anxiety}

\author{
Sara Farahbakhsh Beh', Seyed Abolghasem Mehrinejad²*, Ameneh Moazedian' \\ ${ }^{1}$ Department of Psychology, Semnan Branch, Islamic Azad University, Semnan, Iran \\ ${ }^{2}$ Department of Clinical Psychology, Faculty of Education and Psychology, AlZahra University, Tehran, Iran
}

\section{Article Info:}

\section{ABSTRACT}

Introduction: The quality of life in cancer patients has become increasingly important. The purpose of this study was to examine the predictive model of quality of life in patients with breast cancer based on psychological hardiness, religious orientation, perceived social support, and self-efficacy with mediation of resiliency and death anxiety. Materials and Methods: The research method was analytical and structural equation modeling. The statistical population in this study included all patients with breast cancer referring to Cancer Institute of Imam Khomeini and Milad hospitals in Tehran, Iran in 2018. Purposeful sampling method was used to select the sample. 300 patients with breast cancer were selected based on the criteria for entering and leaving criteria. The research tool was a general self-efficacy questionnaire of Sherer et al, multidimensional perceived social support questionnaire Zamet et al., Allport Religious Orientation Questionnaire, Kobasa Psychological Hardiness Questionnaire, Templar Death Anxiety Scale, The Conor-Davidson Resilience Questionnaire, and the World Health Organization Quality of Life Scale. Results: The results of the model analysis showed that the factor structure of the research scales was acceptable. The results of the conceptual model test showed that the fitting indices of the research model are in a favorable condition. Religious orientation and psychological hardiness on quality of life in patients with breast cancer have significant direct and indirect effects on resiliency and death anxiety. In addition, perceived social support and self-efficacy exhibits a significant indirect effect on death anxiety of patients. Furthermore, resiliency and death anxiety have a significant direct effect on the quality of life of patients with breast cancer.

\section{Key words:}

1. Quality of Life

2. Anxiety

3. Patients religious orientation, and perceived social support in improvement of the quality of life of patients with breast cancer.

*Corresponding Author: Seyed Abolghasem Mehrinejad

E-mail: ab_mehrinejad@yahoo.com 


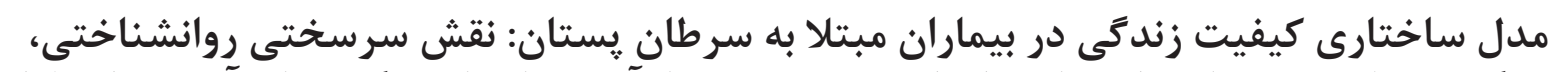

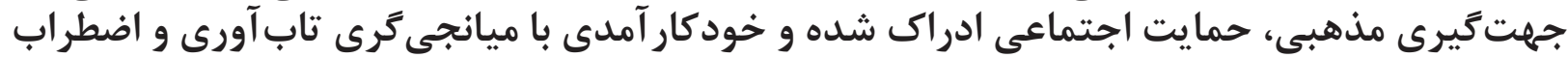
مرك

سارا فرحبخش ببه'، سيد ابوالقاسم مهرى نزاد זّ، آمنه معاضديان'

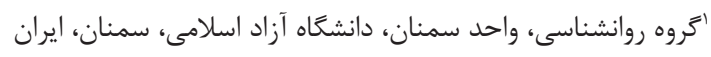

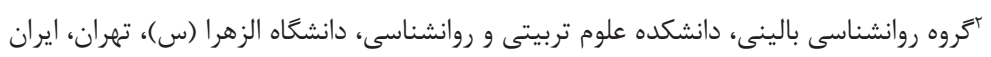

اطلاعات مقاله:

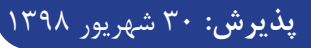

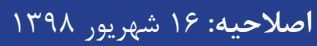

دريافت: ه خرداد هوسا

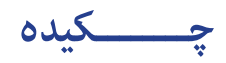

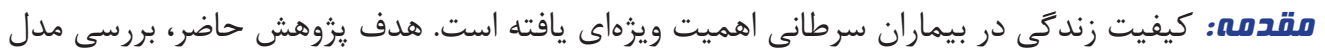

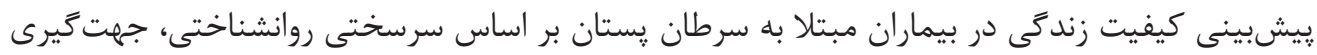

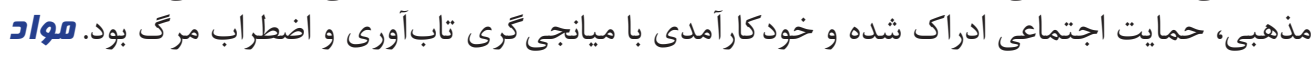

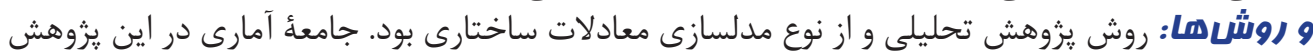

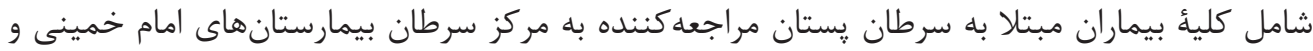

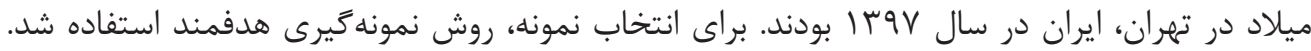

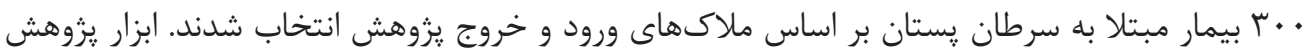

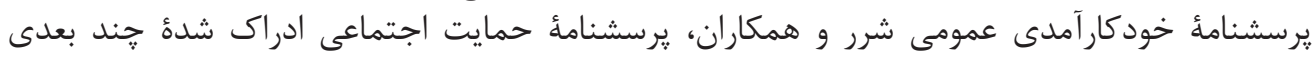

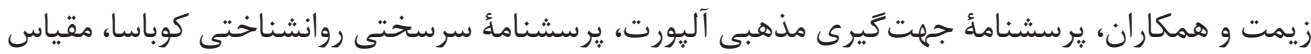

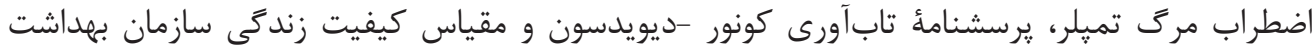

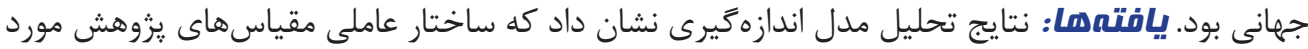

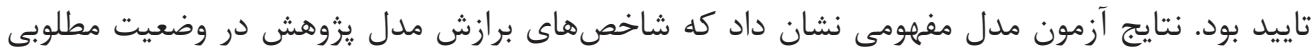

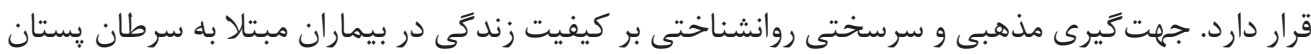

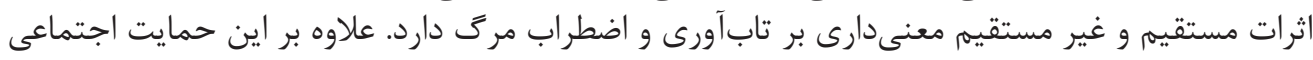

: بليد وازمها:

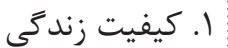

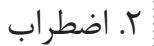
ب: بيماران

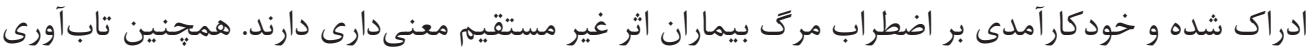

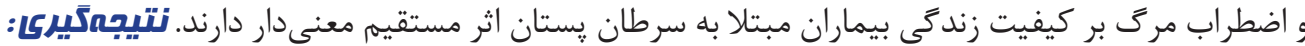

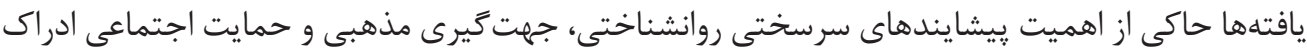

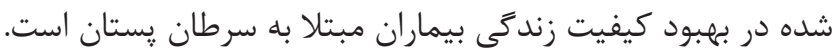




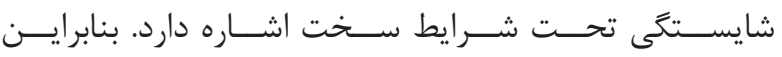

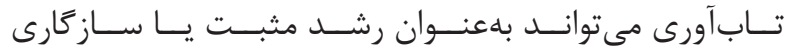

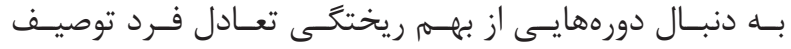

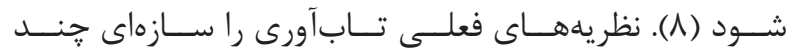

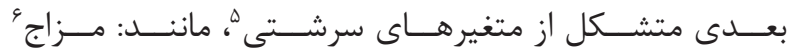

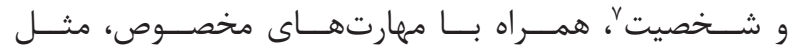

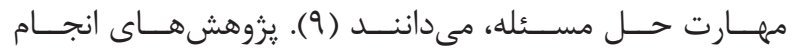

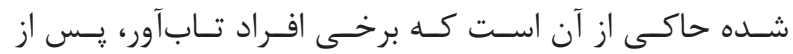

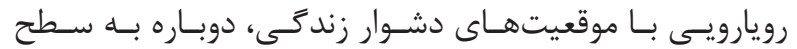

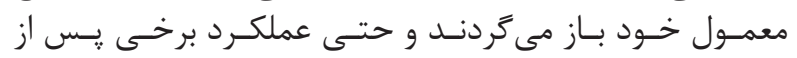

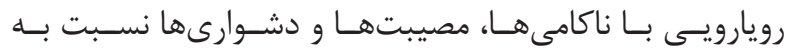

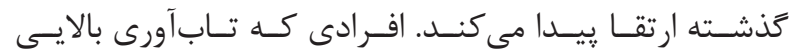

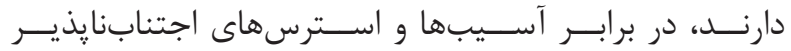

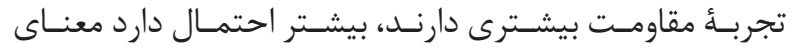

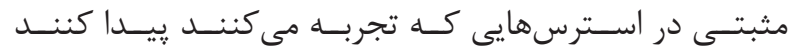

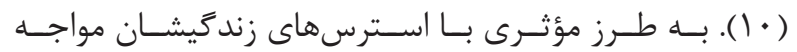

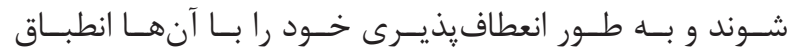

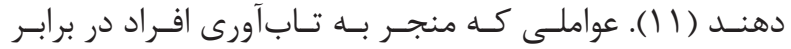

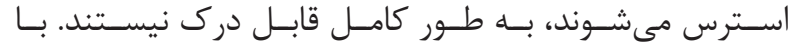

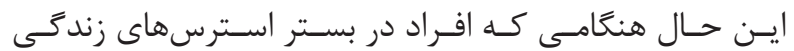

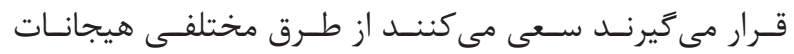

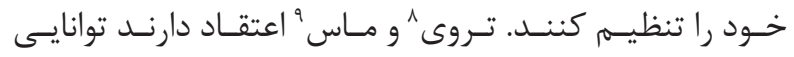

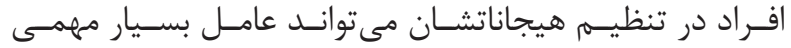

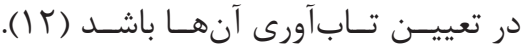

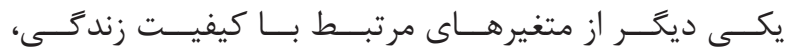

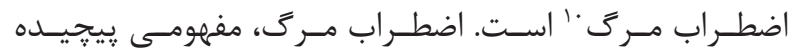

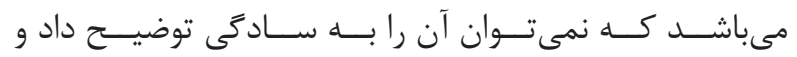

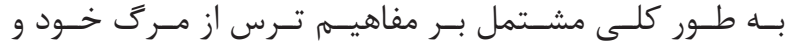

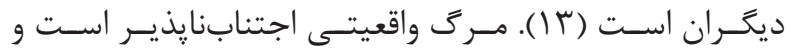

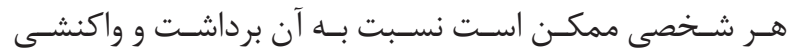

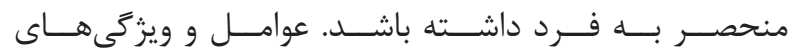

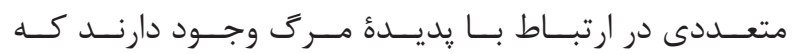

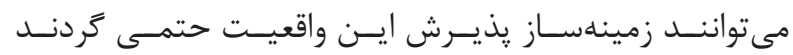

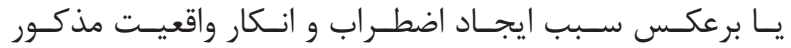

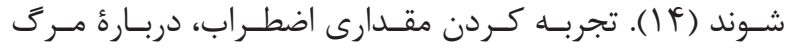

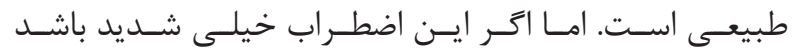

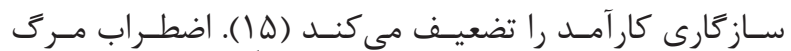

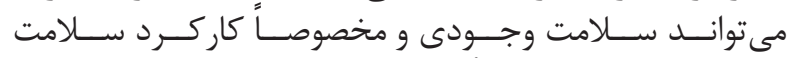

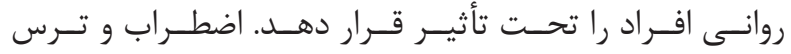

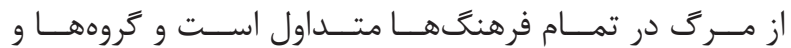

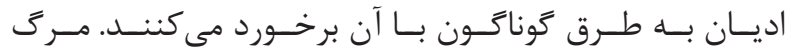

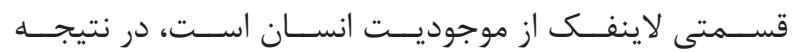

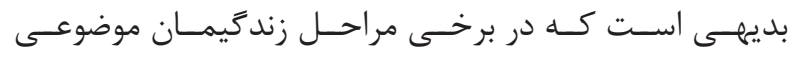

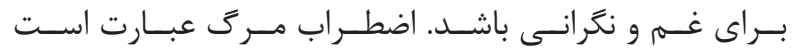

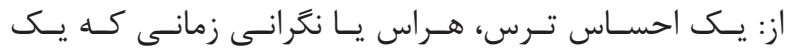

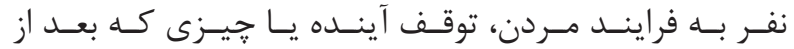

${ }^{1}$ Breast cancer

${ }^{2}$ Quality of life

${ }^{3}$ World health organization

${ }^{4}$ Resilience

${ }^{5}$ Constitutional
مقدمه

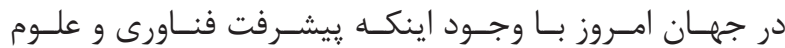

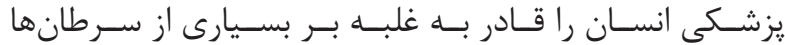

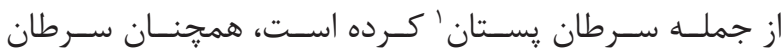

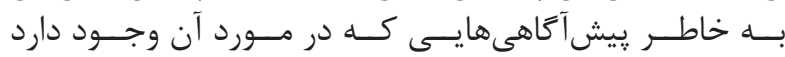

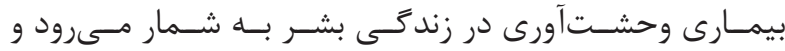

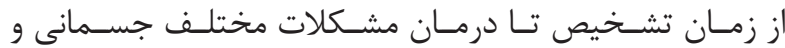

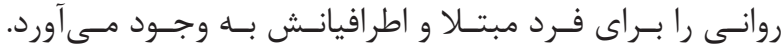

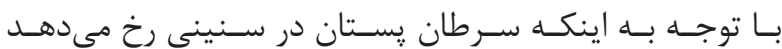

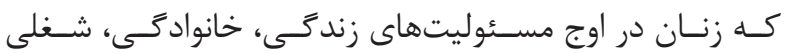

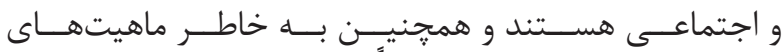

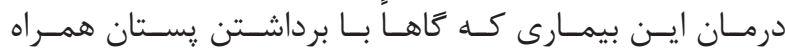

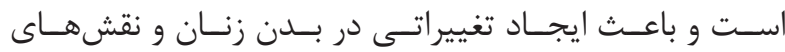

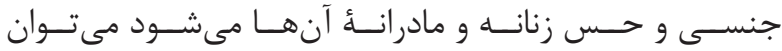

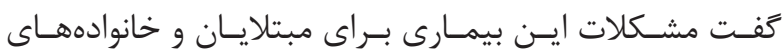

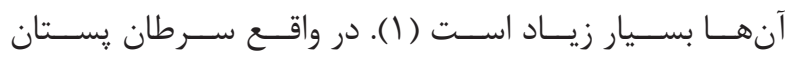

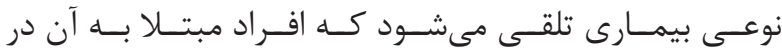

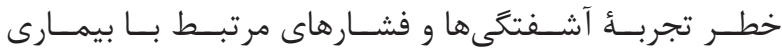

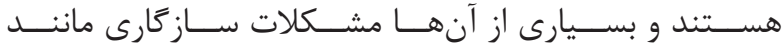

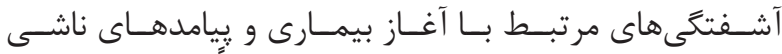

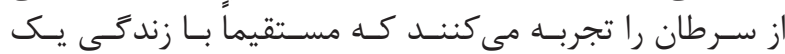

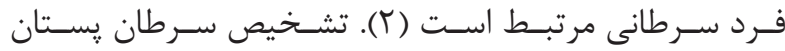

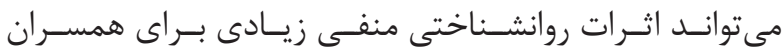

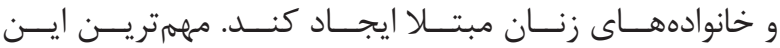

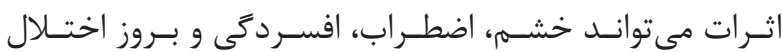

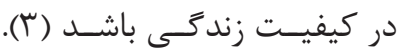

طـى سـال هاى اخيـر بررسـى كيفيـت زندَّى بـ در مبتلايــان

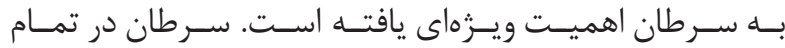

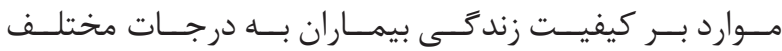

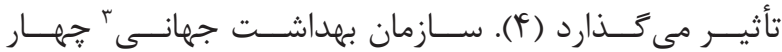

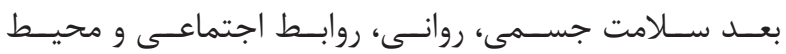

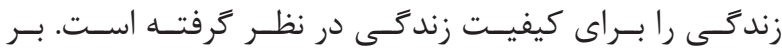

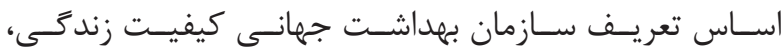

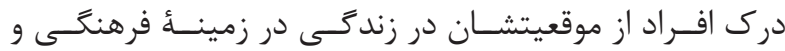

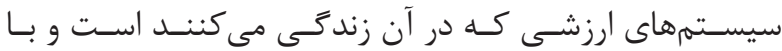

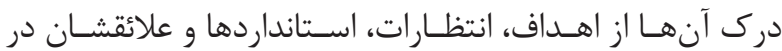

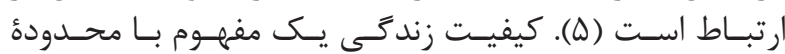

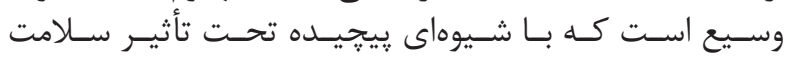

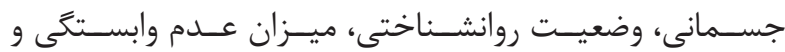

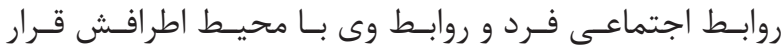

دارد (9) (9)

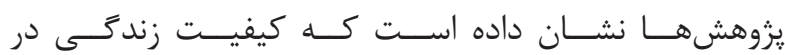

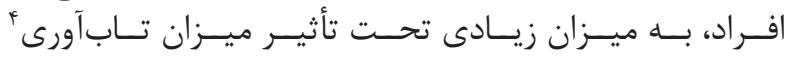

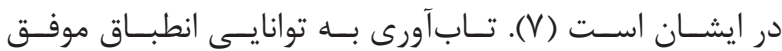

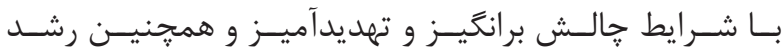

\footnotetext{
${ }^{6}$ Temperament

${ }^{7}$ Personality

${ }^{8}$ Troy

${ }^{9}$ Mauss

${ }^{10}$ Death anxiety
} 


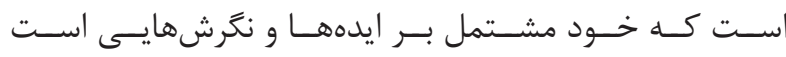

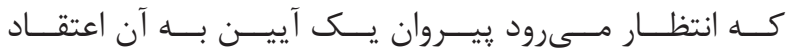

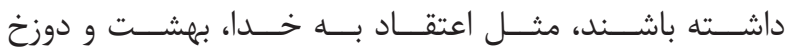

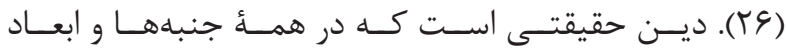

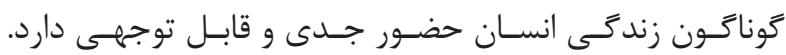

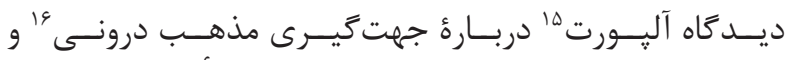

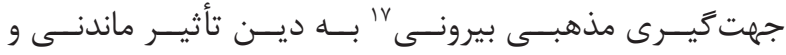

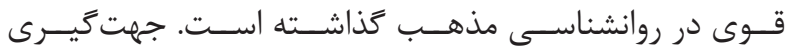

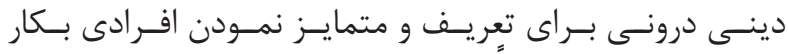

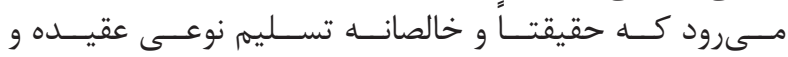

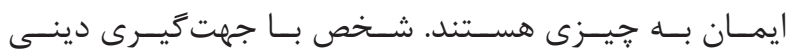

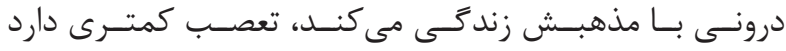

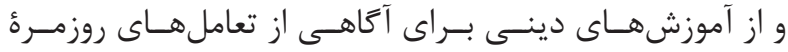

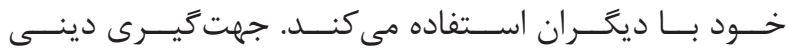

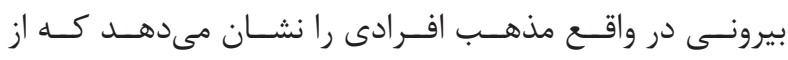

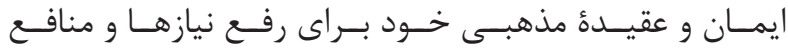

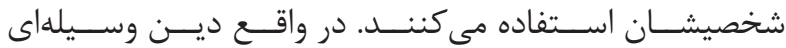

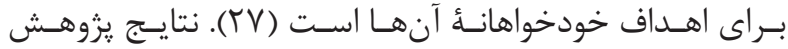

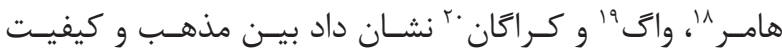

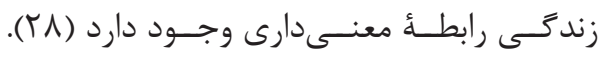

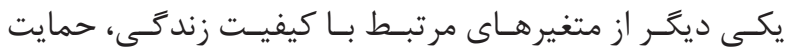

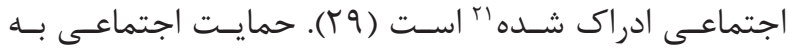

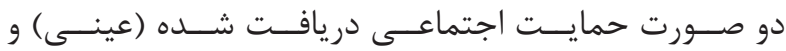

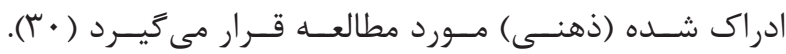

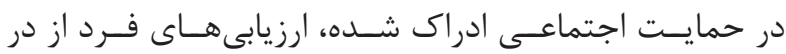

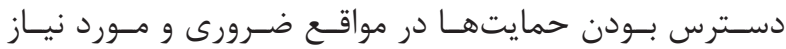

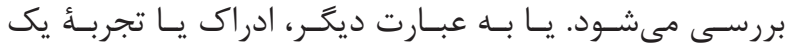

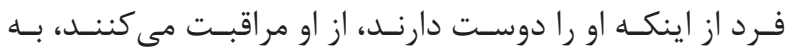

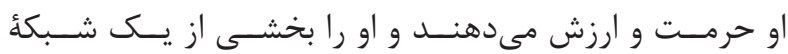

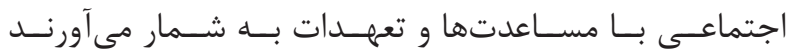

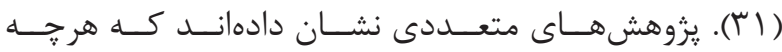

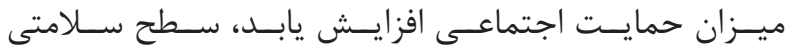

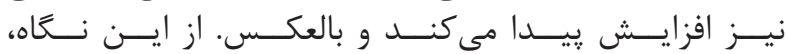

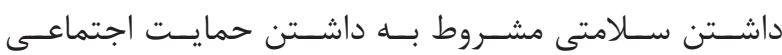

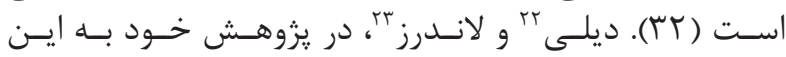

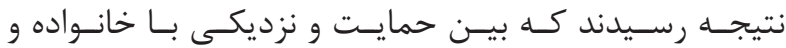

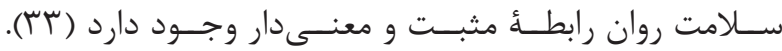

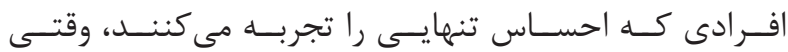

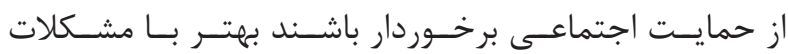

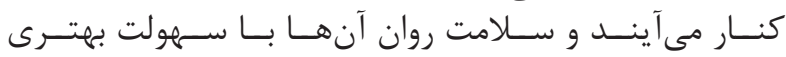

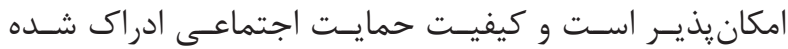

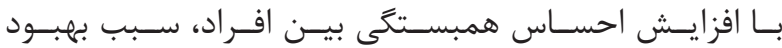

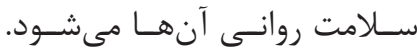

${ }^{11}$ Belskey

${ }^{12}$ Psychological hardiness

${ }^{13}$ Kobasa

${ }^{14}$ Religious

${ }^{15}$ Alport

${ }^{16}$ Internal religious orientation

${ }^{17}$ Outer religious orientation

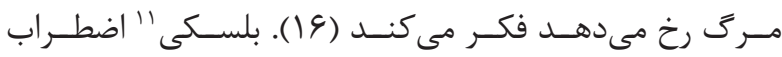

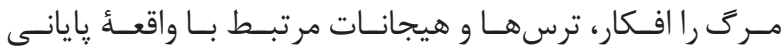

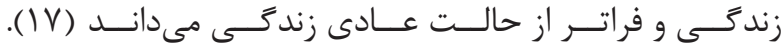

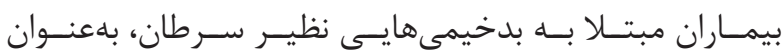

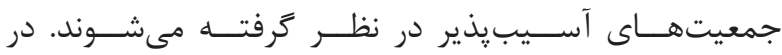

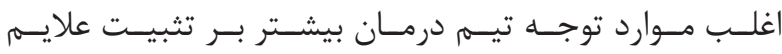

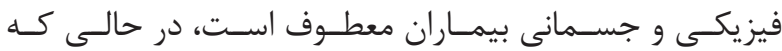

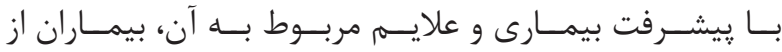

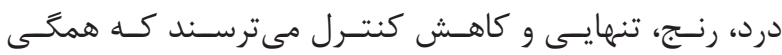

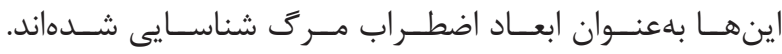

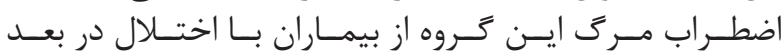

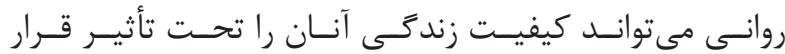

دهـد (1) (1).

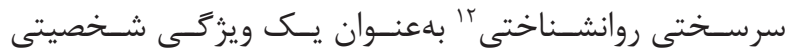

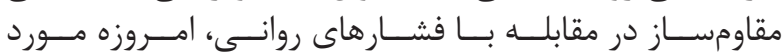

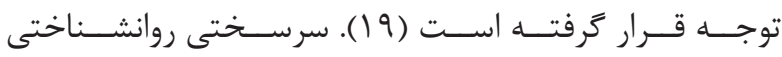

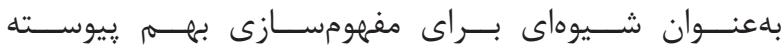

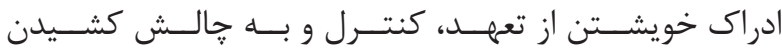

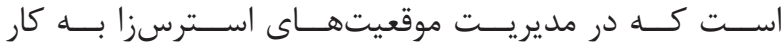

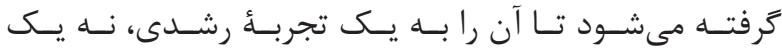

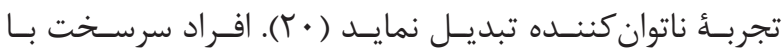

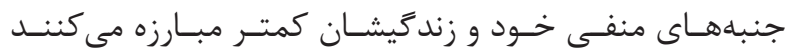

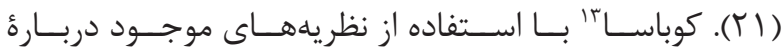

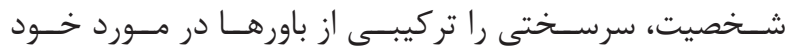

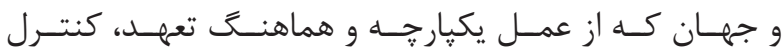

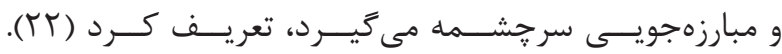

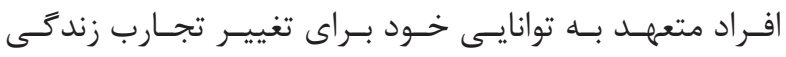

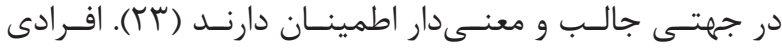

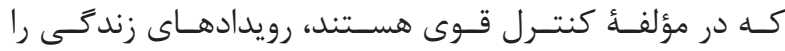

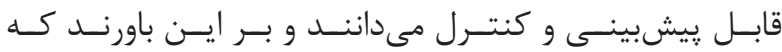

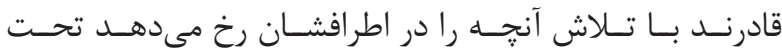

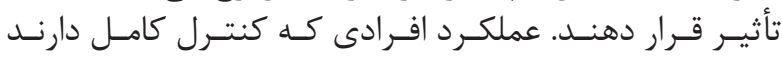

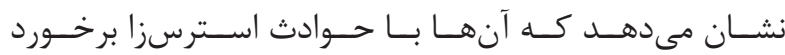

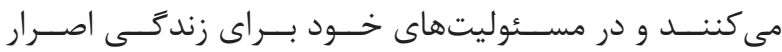

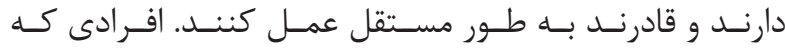

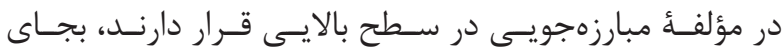

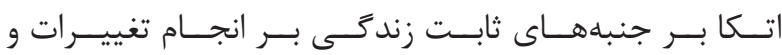

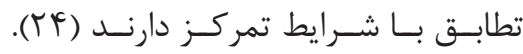

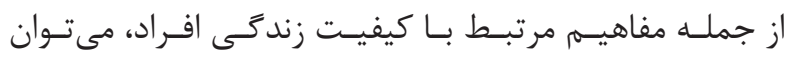

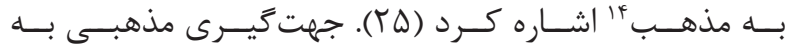

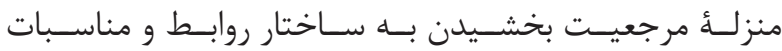

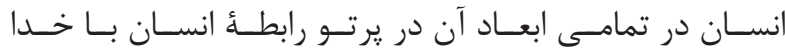

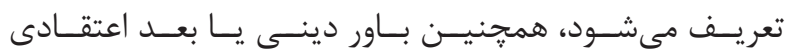

\footnotetext{
${ }^{18}$ Hammer

${ }^{19}$ Wade

${ }^{20}$ Cragun

${ }^{21}$ Perceived social support

${ }^{22}$ Dehle

${ }^{23}$ Landers
} 


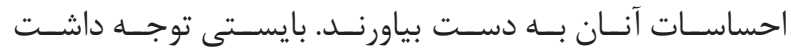

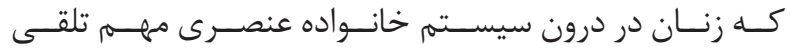

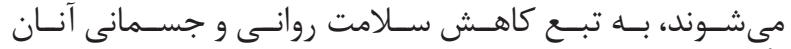

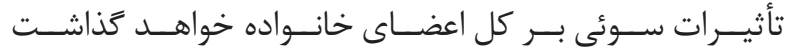

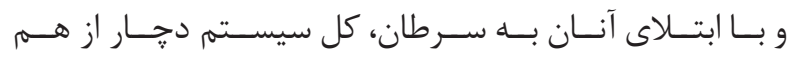

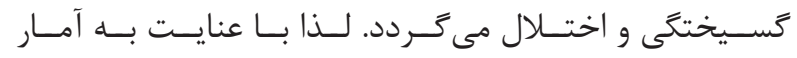

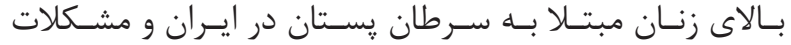

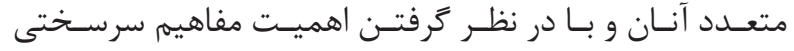

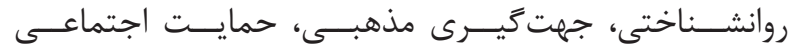

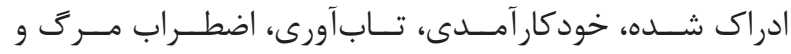

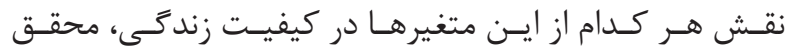

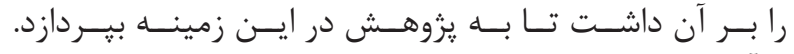

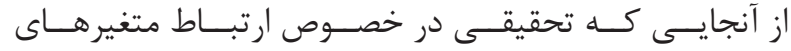

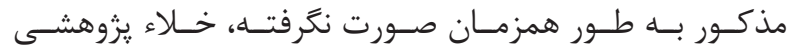

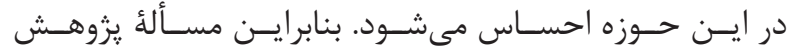

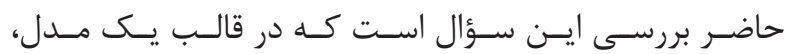

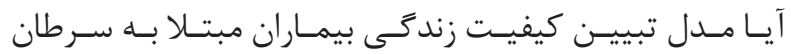

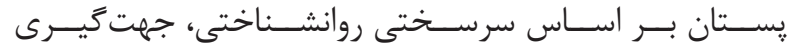

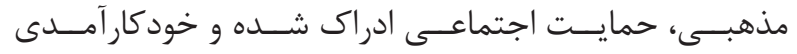

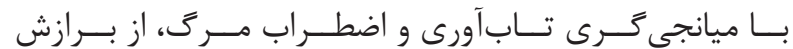

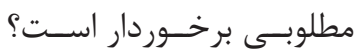

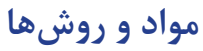

مقياس كيفيت زندىى

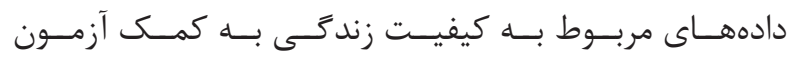

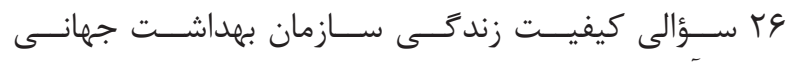

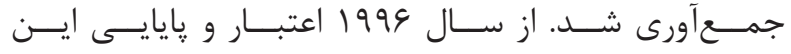

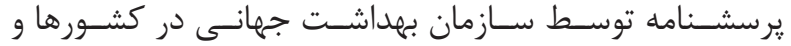

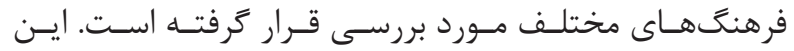

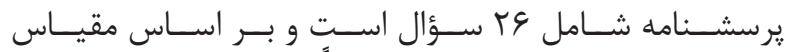

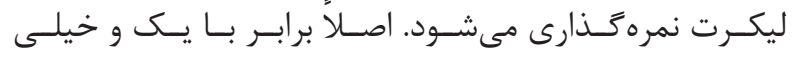

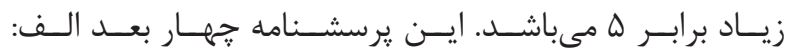

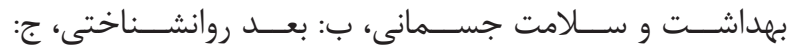

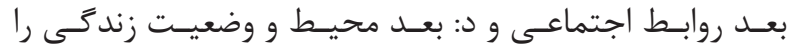

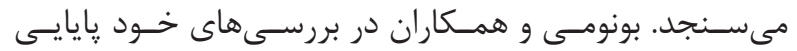

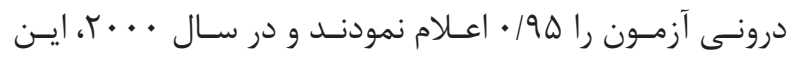

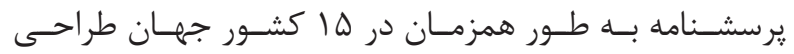

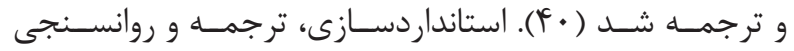

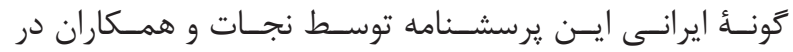

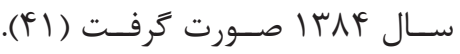

$$
\text { يرسشنامة تاب آورى كونور -ديويدسون }
$$

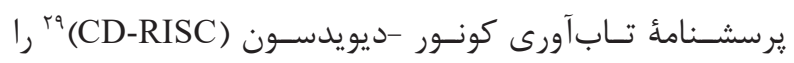

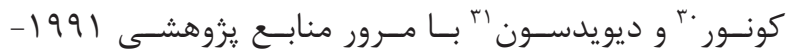

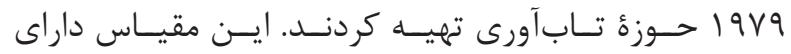

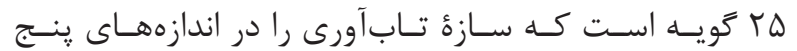

\footnotetext{
${ }^{24}$ Self-efficacy

${ }^{25}$ Bandura

${ }^{26}$ Herts

${ }^{27}$ Khaled
}

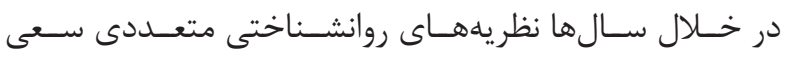

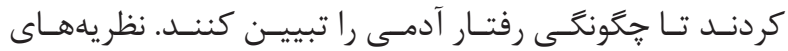

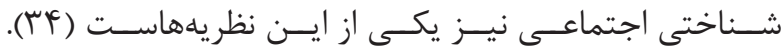

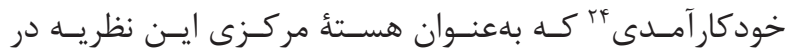

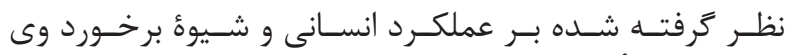

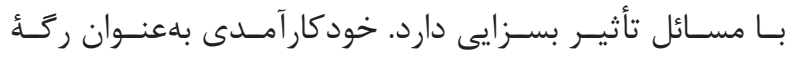

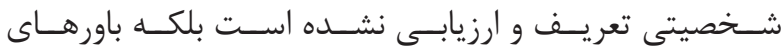

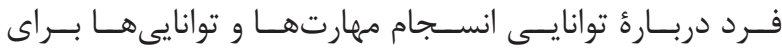

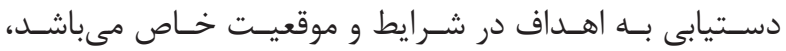

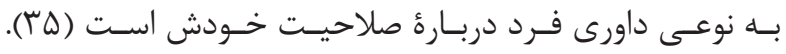

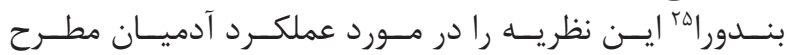

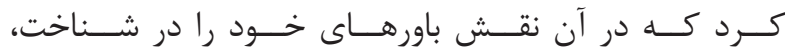

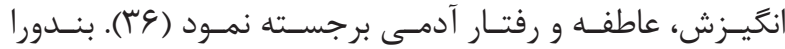

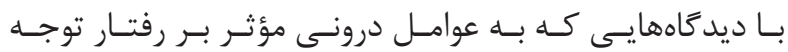

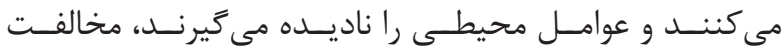

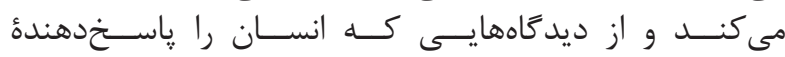

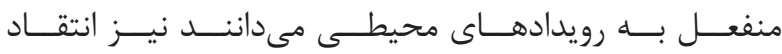

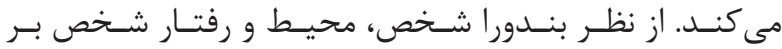

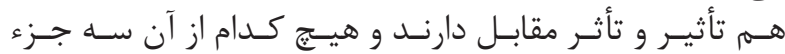

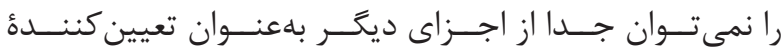

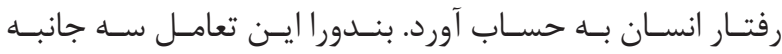

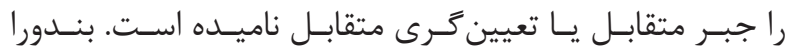

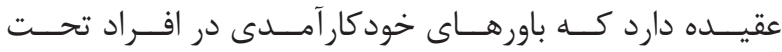

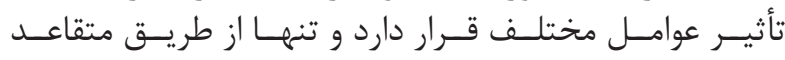

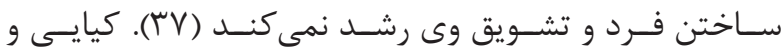

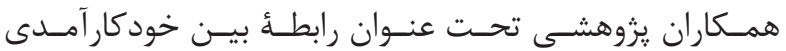

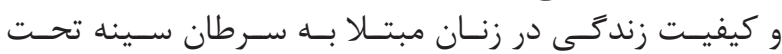

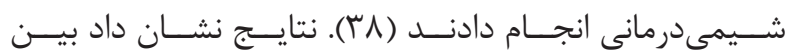

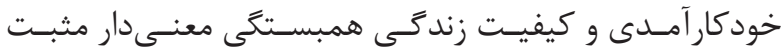

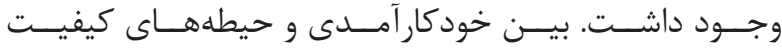

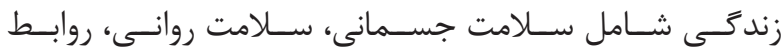

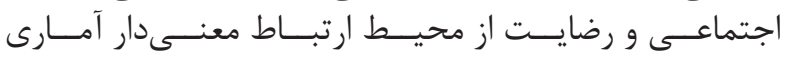

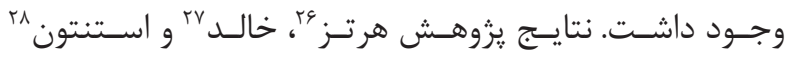

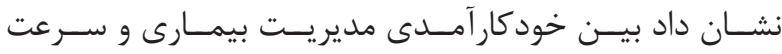

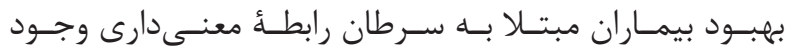

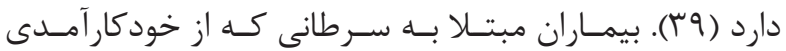

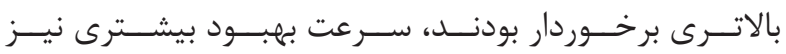
داشـتند.

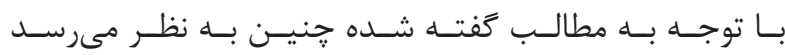

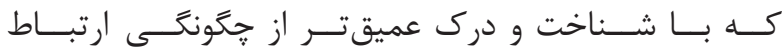

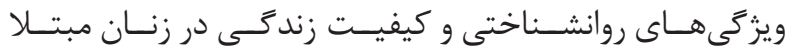

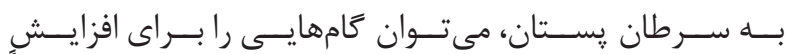

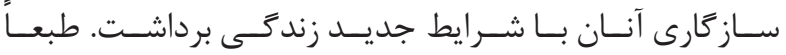

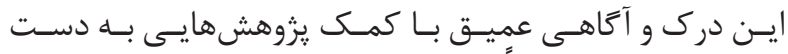

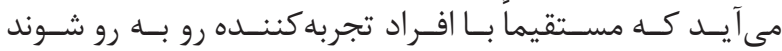

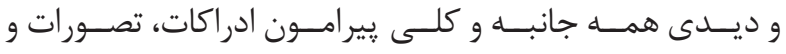

\section{${ }^{28}$ Stanton}

${ }^{29}$ Connor-davidson resilience scale

${ }^{30}$ Connor

${ }^{31}$ Davidson 
برسشنامة جههت تيرى مذهبى

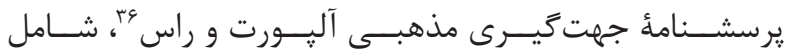

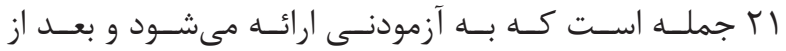

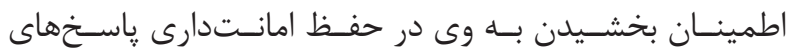

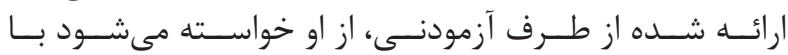

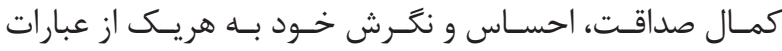

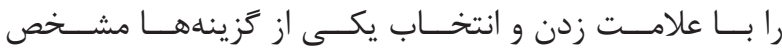

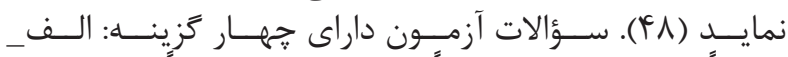

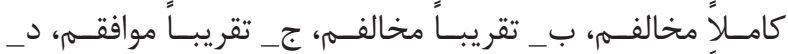

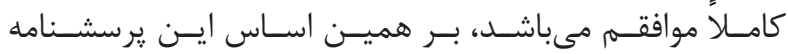

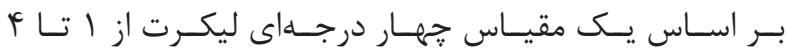

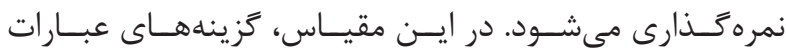

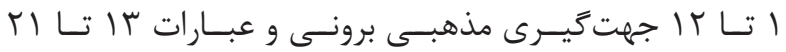

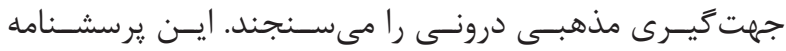

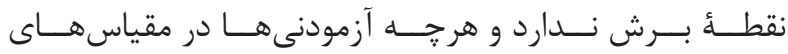

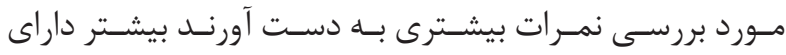

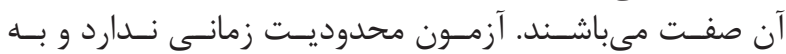

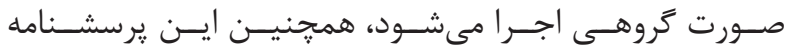

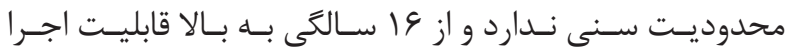

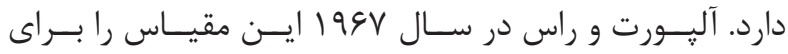

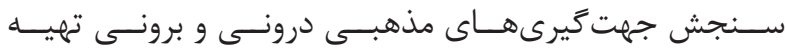

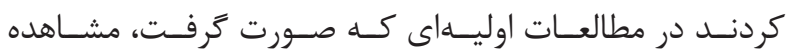

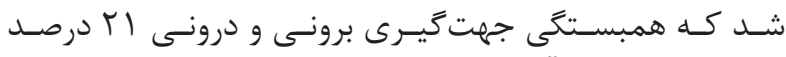

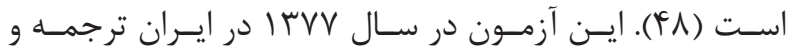

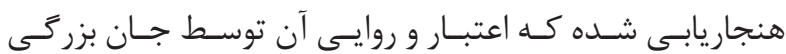

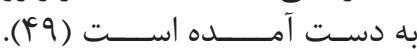

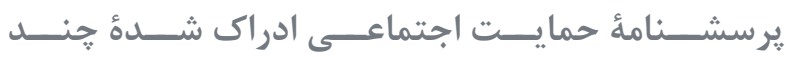

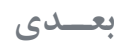

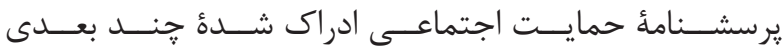
(MSPSS)

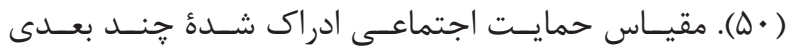

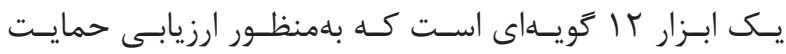

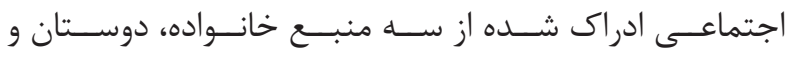

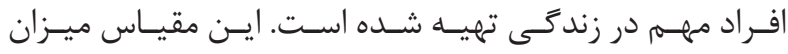

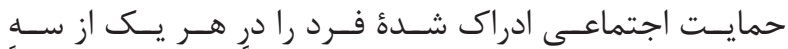

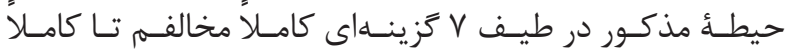

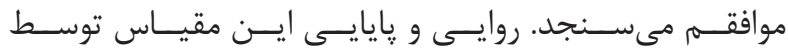

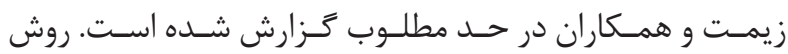

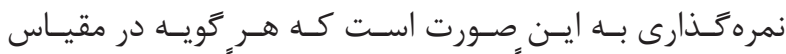

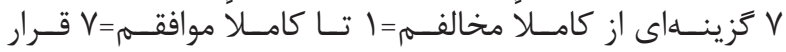

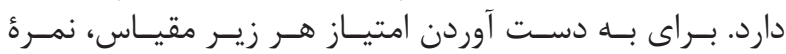

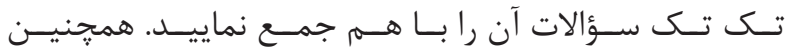

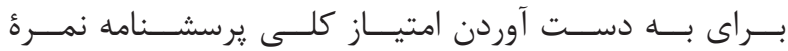

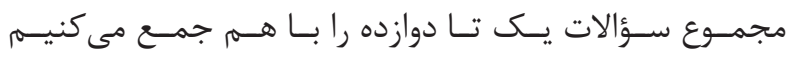

\section{${ }^{32}$ Templer}

${ }^{33}$ Sayino

${ }^{34}$ Kelaien

${ }^{35}$ Kobasa.'S hardiness questionnaire

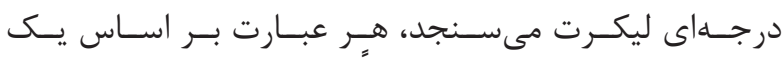

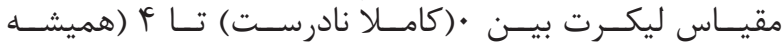

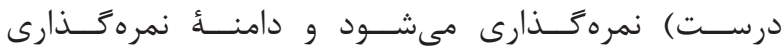

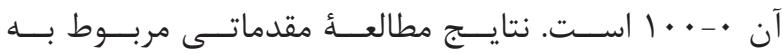

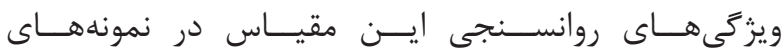

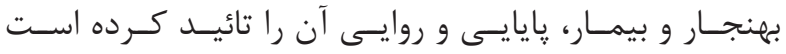

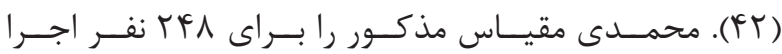

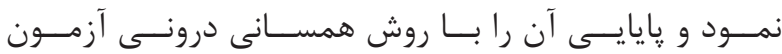

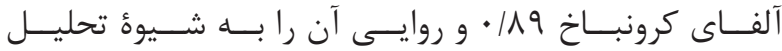

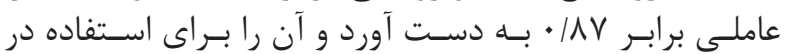

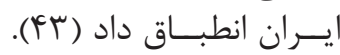

$$
\text { مقياس اضطر اب مرى }
$$

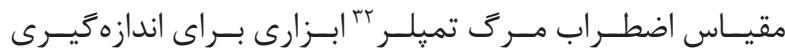

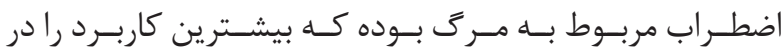

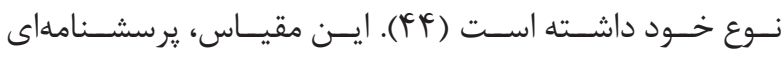

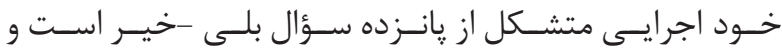

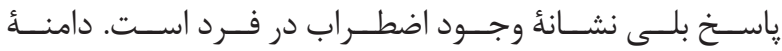

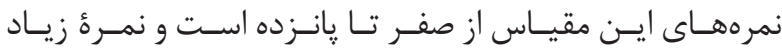

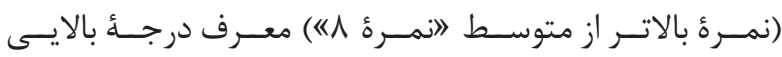

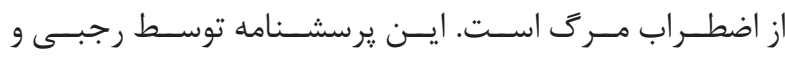

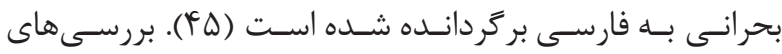

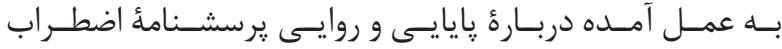

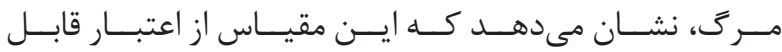

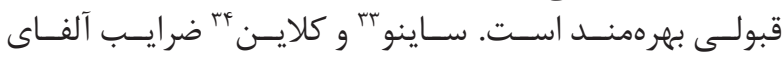

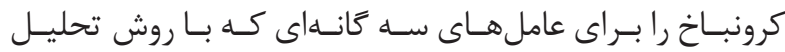

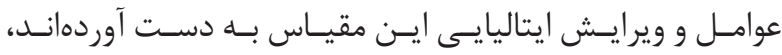

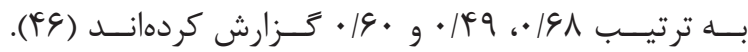

يرسشنامةٔ سرسختى روانشناختى كوباسا (19V9)

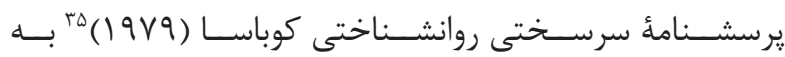

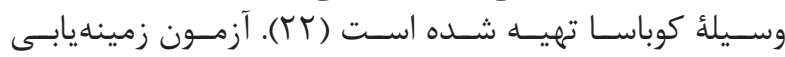

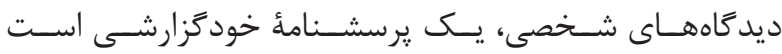

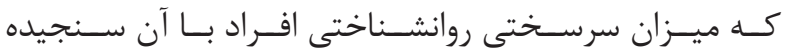

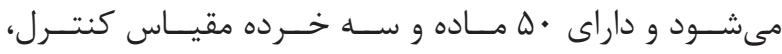

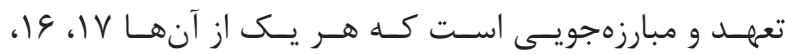

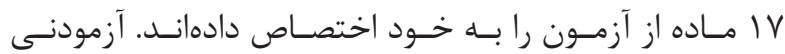

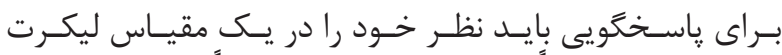

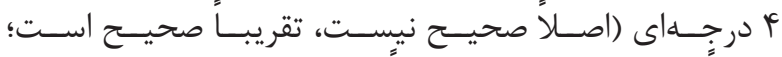

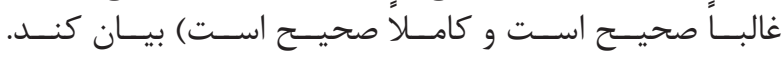

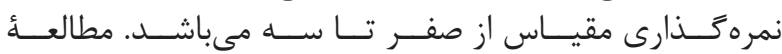

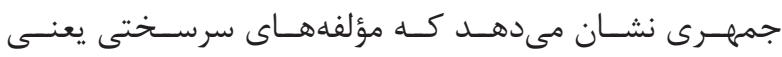

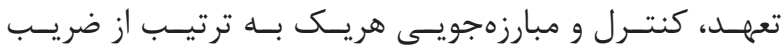

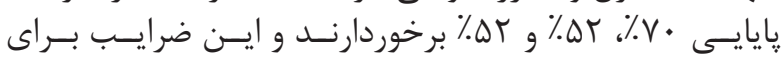

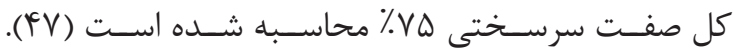

\footnotetext{
${ }^{36}$ Ross

${ }^{37}$ Multidimensional scale of perceived social support ${ }^{38}$ Zimet
} 


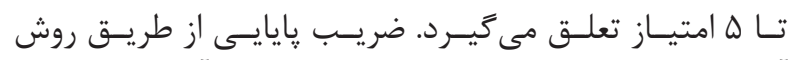

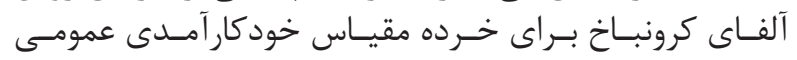

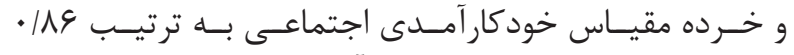

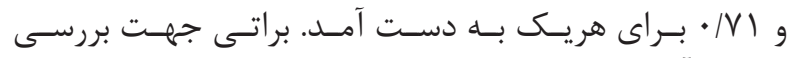

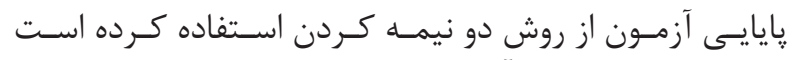

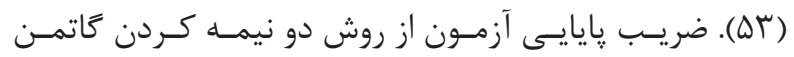

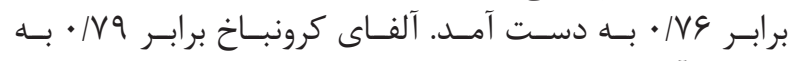

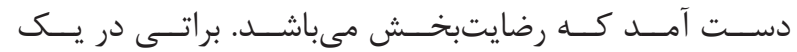

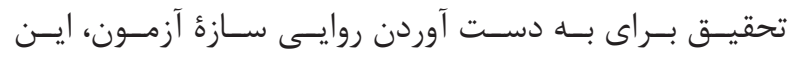

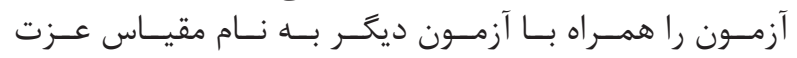

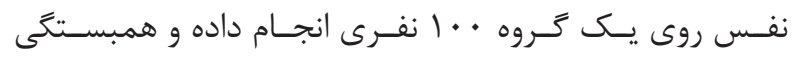

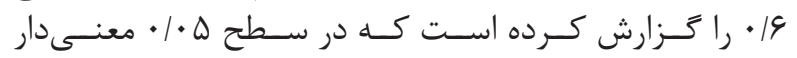

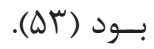

تجريه و تحليل دادهها

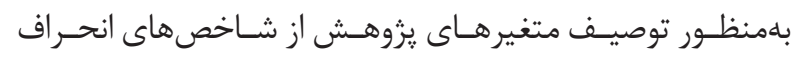

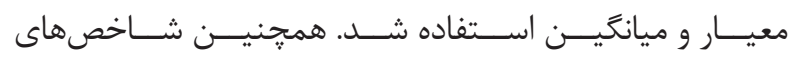

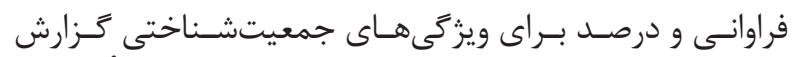

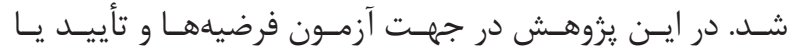

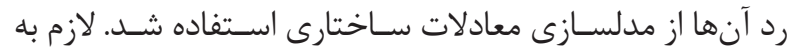

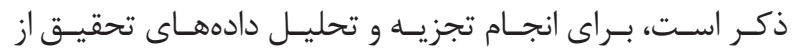

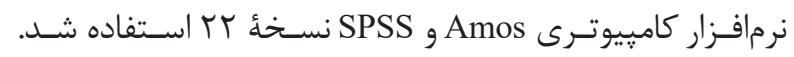

بافته ها

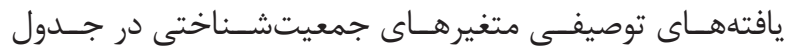

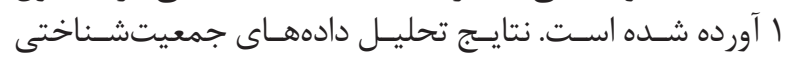

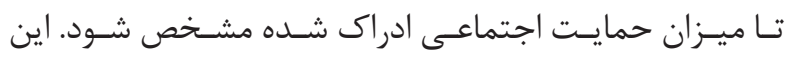

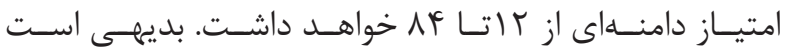

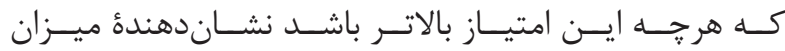

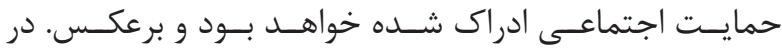

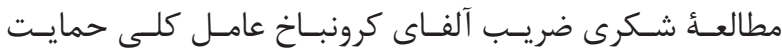

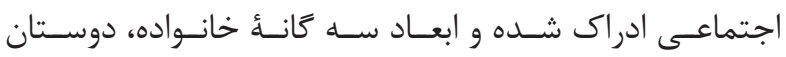

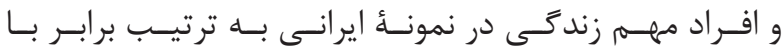
/ 99

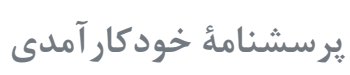

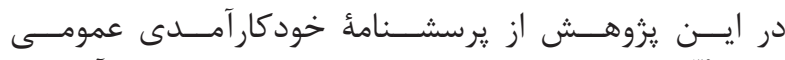

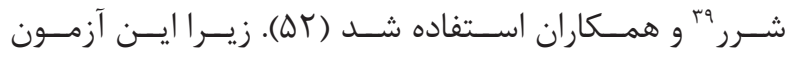

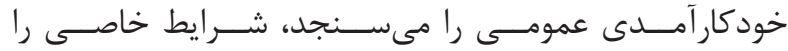

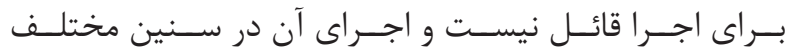

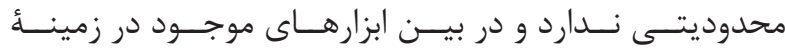

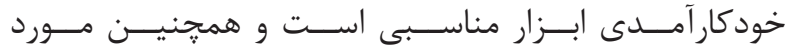

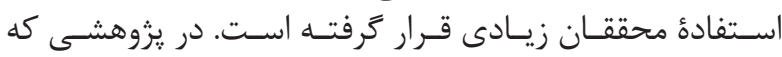

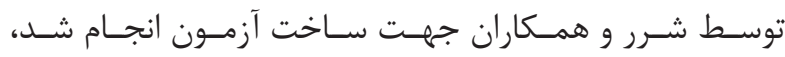

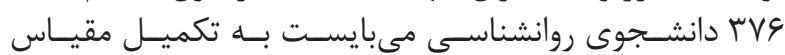

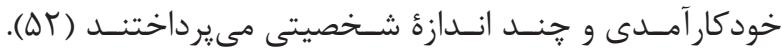

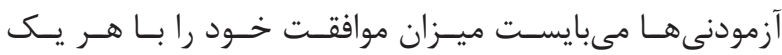

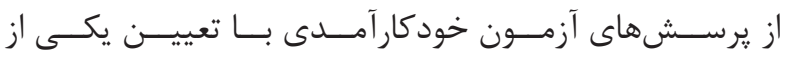

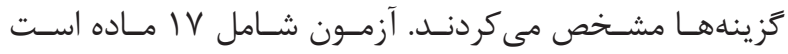

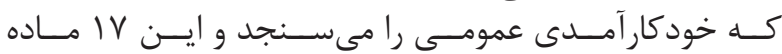

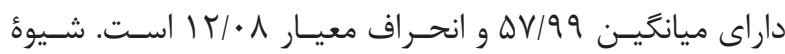

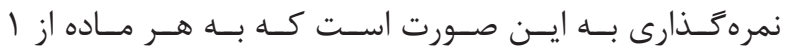

\begin{tabular}{|c|c|c|c|c|c|}
\hline انحراف استاندارد & ميانكين & درصد & فراوانى & شاخص آمارى & متغير \\
\hline \multirow{4}{*}{$\Delta / \Delta \varphi$} & \multirow{4}{*}{ FN/GT } & $r \Delta / r r$ & ve & 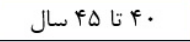 & \multirow{4}{*}{ سن سن } \\
\hline & & $r f$ & $1 . r$ & عز تا •ه سال & \\
\hline & & $r Y / 9 V$ & $8 \Lambda$ & ا ه تا ه هال سال & \\
\hline & & 11 & $\Delta f$ & عه تا •ع سال & \\
\hline \multirow{4}{*}{ - } & \multirow{4}{*}{-} & $r q / ६ \mathrm{~V}$ & 119 & ديبله & \multirow{5}{*}{ تحصيلات } \\
\hline & & rr & 89 & فوق ديبلم & \\
\hline & & rq & $\wedge \vee$ & ليسانس & \\
\hline & & N/Tr & ro & فوق ليسانس و بالاتر & \\
\hline- & - & $1 \ldots$ & $r \cdot \cdot$ & مجموع كل & \\
\hline
\end{tabular}

جدول r - يافتهاى توصيفى خر دمقياسهاى تحقيق.

\begin{tabular}{|c|c|c|}
\hline انحراف استاندارد & ميانكين & شاخص آمارى \\
\hline $11 / 4 V$ & $9 \vee / 11$ & سرسختى روانشناختى \\
\hline R/IT & $r V / \Lambda$ & جهت كيرى مذهبى بيرونى \\
\hline$V / r \cdot$ & $r V / \Delta V$ & جهت تحيرى مذهبى درونى \\
\hline$N / / \Delta$ & $f \cdot / 48$ & خودكار آمدى \\
\hline $1 \cdot|8|$ & $f \cdot / 09$ & حمايت اجتماعى ادراك شده \\
\hline V/r人 & $f N / \cdot r$ & تابآورى \\
\hline$\Delta / \backslash \wedge$ & I T/A & اضطراب مركى \\
\hline $\mid f / f 8$ & ^१/१. & كيفيت زندكى \\
\hline
\end{tabular}




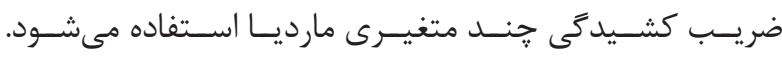

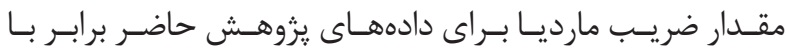

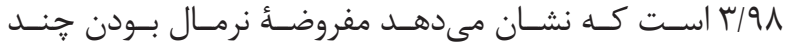

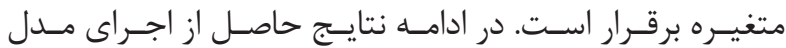

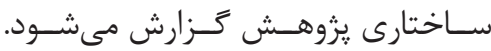

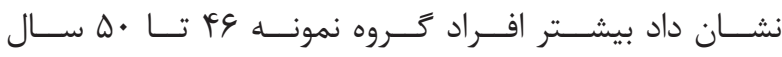

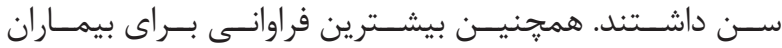

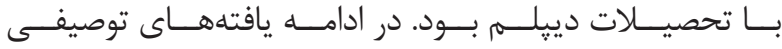

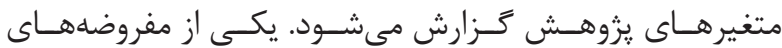

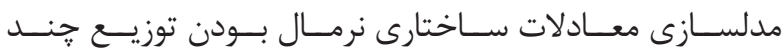

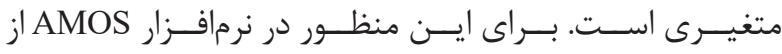

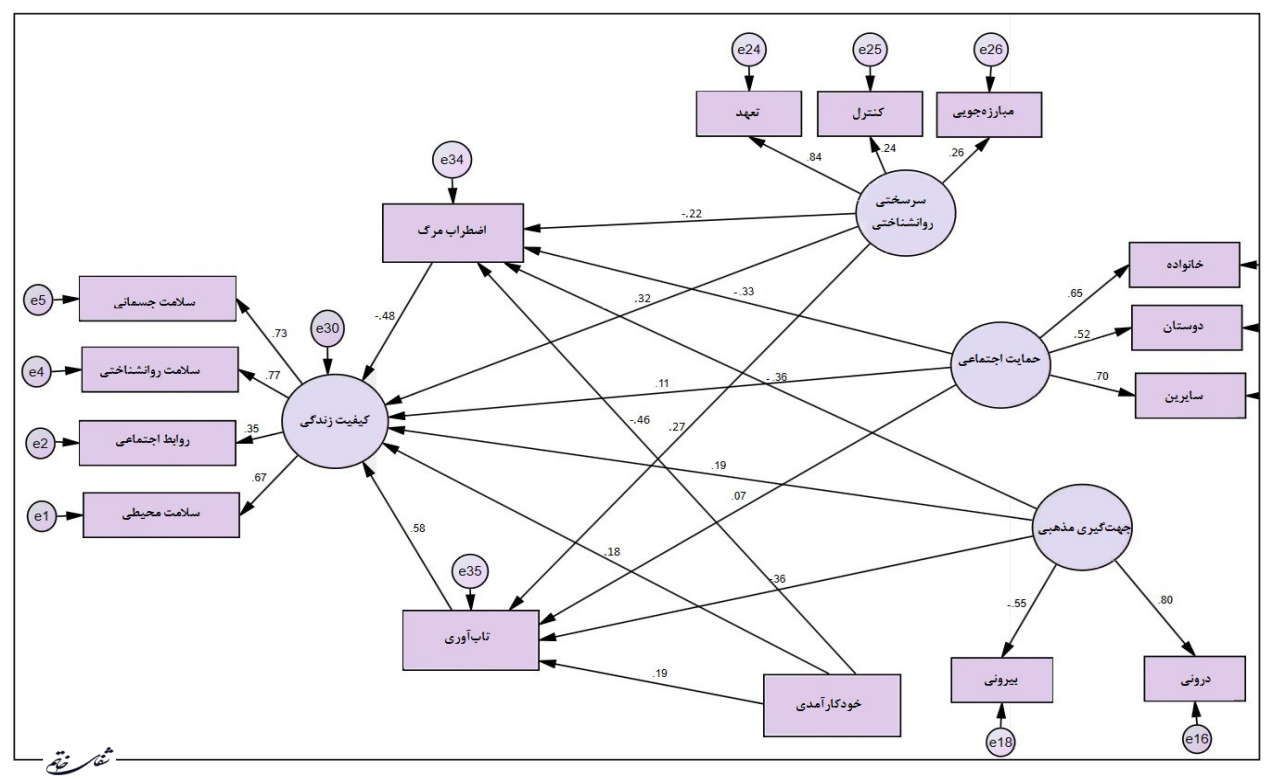

تصوير ا- مدل در حالت ضرايب استاندارد شده.

\begin{tabular}{|c|c|c|}
\hline \multicolumn{2}{|c|}{ شاخصهاى برازش } & \multirow{2}{*}{ 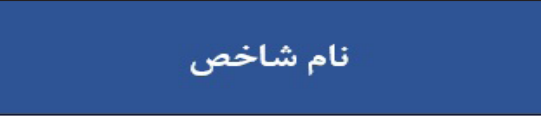 } \\
\hline حد مجاز & مقدار & \\
\hline 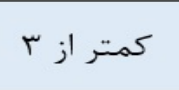 & $1 / 19$ & $\frac{\mathrm{x}^{2}}{\mathrm{df}}$ \\
\hline 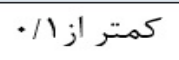 & $\cdot / \cdot F$ & RMSEA (ريشه ميانخين خطاى برآورد) \\
\hline 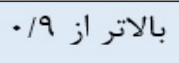 & $\cdot / 9 \mathrm{~V}$ & CFI \\
\hline 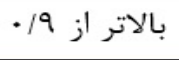 & $\cdot / 90$ & NFI \\
\hline 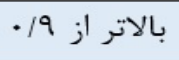 & $\cdot / 91$ & GFI \\
\hline بالاتر از 9/9. & $\cdot / 9 \Delta$ & (نيكويى برازشاصلاح شده) AGFI \\
\hline
\end{tabular}

جدول F- ضرايب و معنى دارى اثرات مستقيم و غير مستقيم بر كيفيت زندگى.

\begin{tabular}{|c|c|c|c|c|c|c|}
\hline معنى دارى & آمارة معنى دارى & B B استاندار دشده & ضريب استاندار ن نشده & توع اثر & متغير بيشبين & متغير ملاك \\
\hline$\cdot / \cdot \cdot 1$ & $r / \Delta \Lambda$ & $\cdot / 4 f$ & $\cdot / 4 t$ & مستقيم & سرسختى روانشثاختى & كيفيت زندئى \\
\hline 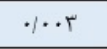 & T/QT &.$/ 19$ & .119 & به واسطذ ثابآورى & سرسختى روانشثاختى & كيفيت زندكى \\
\hline .1 .9 & $1 / \Delta 9$ & $\cdot / 11$ & ז & به واسطئ اضطراب مركى & سرسختى روانشتاختى & كيفيت زندثى \\
\hline$\cdot \cdot \cdot r$ & $T / \cdot F$ & .119 & .1 .9 & مستقيم & جهت كيرى مذهبى & كيفيت زندكى \\
\hline.$/ .4$ & t/IT &.$/ 19$ & .111 & به واسطة اضطراب مركى & جهت كيرى منهبى & كيفيت زندكى \\
\hline$\cdot 1 \cdot 1$ & $T / T \Delta$ & $\cdot(\pi)$ &.$/ 14$ & به واسطة تابآورى & جهت كيرى مذهبى & كيفيت زثدثى \\
\hline$\cdot 1 \cdot f$ & $1 / 91$ &.$/ 11$ & $\cdot \pi \cdot$ & مستقيم & حمايت اجتماعى & كيفيت زندكى \\
\hline$\cdot 1 \cdot 1$ & $r / \cdot 1$ & .110 & .119 & به واسطذ اضطراب مركى & حمايت اجتماعى & كيفيت زندكى \\
\hline$\cdot / r 9$ & $\cdot / \mathrm{VA}$ & $.1 \cdot 4$ & • & به واسطةُ تابآورى & حمايت اجتماعى & كيفيت زندكى \\
\hline$\% \cdot \cdots 1$ & $7 / .9$ &.$/ 11$ & $\cdot \pi \cdot$ & مستقيمي & خودكارأهدى & كيفيت زندكى \\
\hline . & $1 / \cdot r$ & .1 .9 & $\cdot \cdot v$ & به واسطةُ تابآورى & خود كارآمدى & كيفيت زندكى \\
\hline$\%$. r & r/G & .119 &.$/ 1 \mathrm{~V}$ & به واسطة اضطراب مركى & خود كارآمدى & كيفيت زندكّى \\
\hline$\cdot / \cdot 1$ & $\mathrm{~V} / 19$ & $\cdot / \Delta \Lambda$ & .94 & مستقيم & تابآورى & كيفيت زندكى \\
\hline$\because \cdots 1$ & -9110 & $-\cdot / 4 A$ & $-\cdot / \Delta T$ & مستقيم & اضطراب مرك & كيفيت زندكى \\
\hline
\end{tabular}




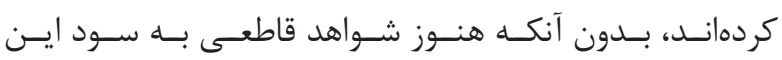

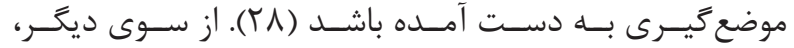

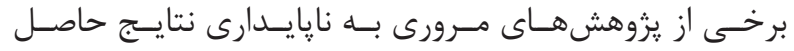

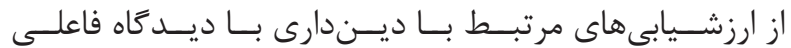

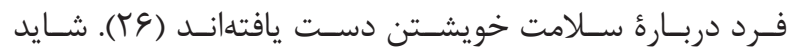

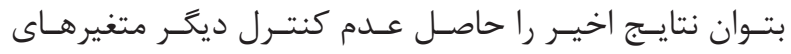

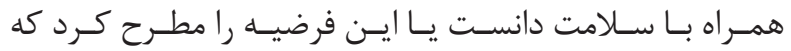

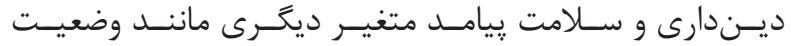

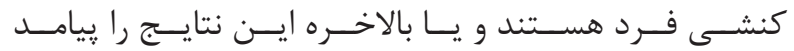

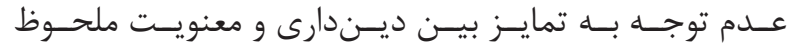

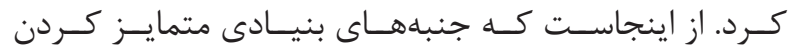

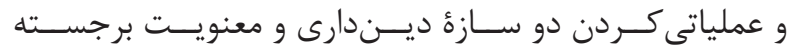

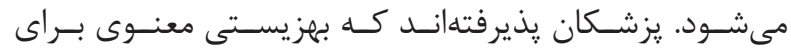

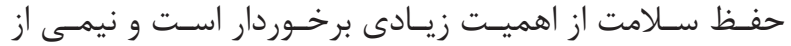

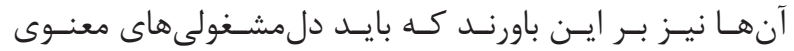

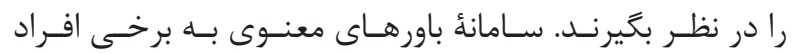

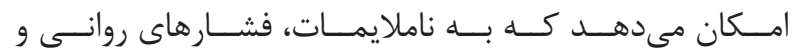

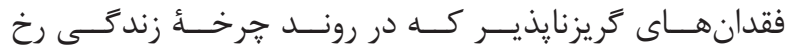

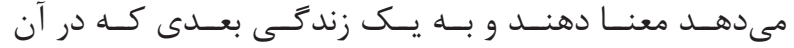

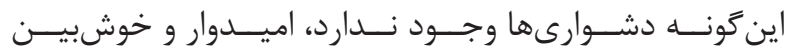

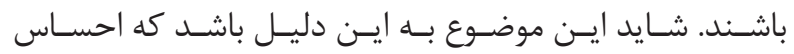

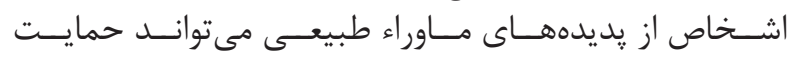

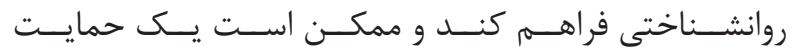

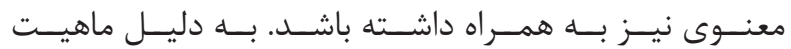

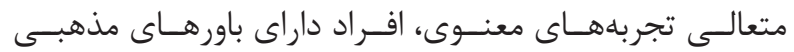

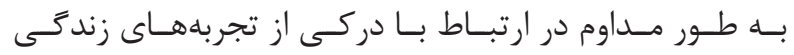

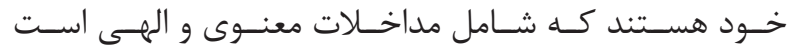

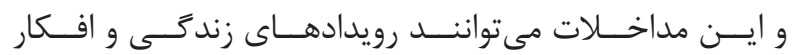

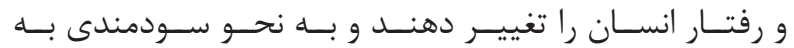

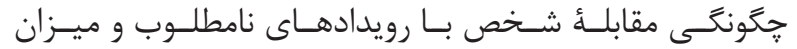

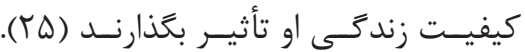

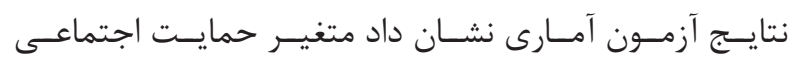

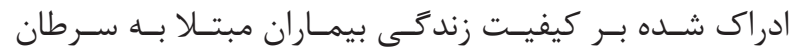

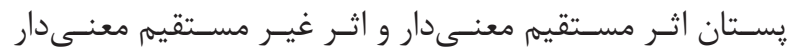

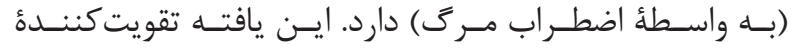

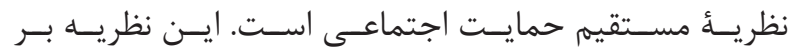

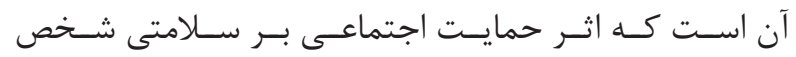

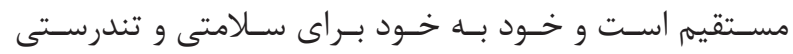

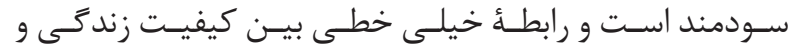

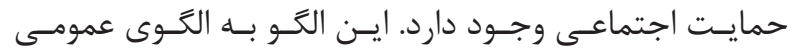

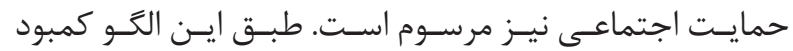

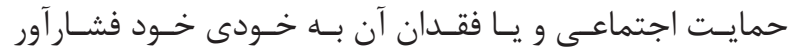

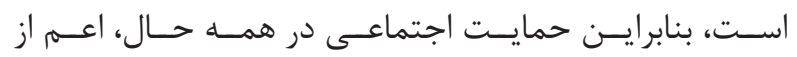

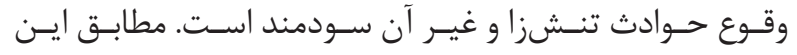

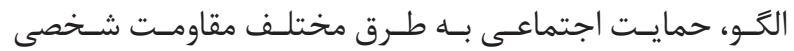

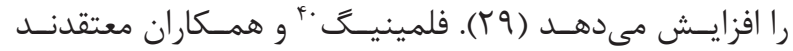

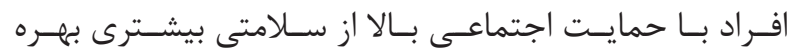

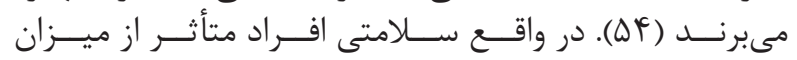

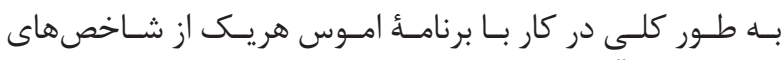

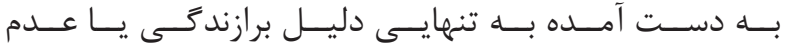

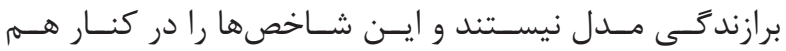

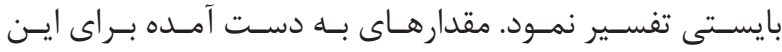

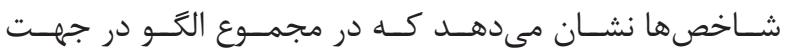

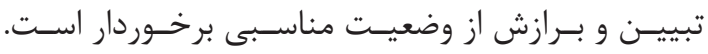

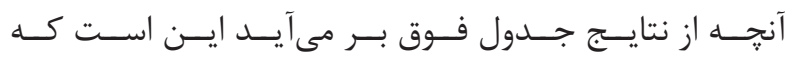

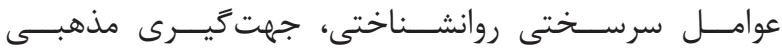

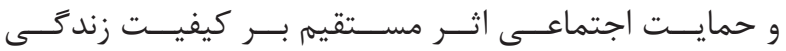

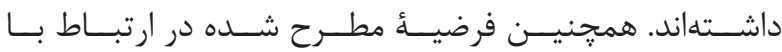

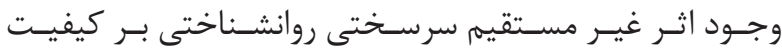

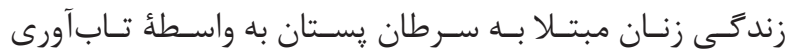

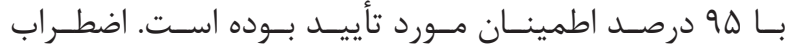

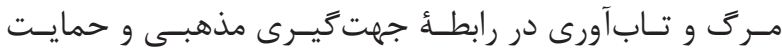

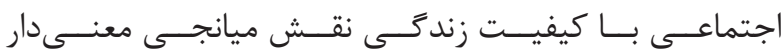

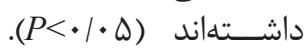

\section{بحث و نتيجه كيرى}

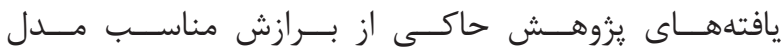

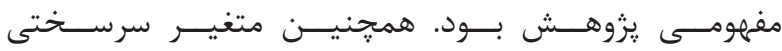

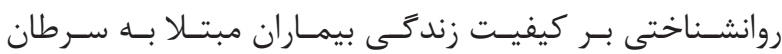

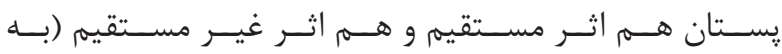

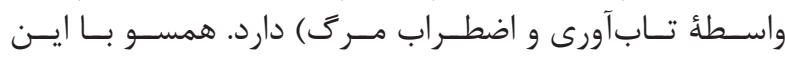

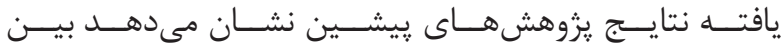

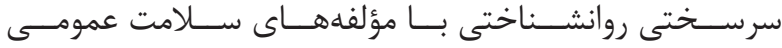

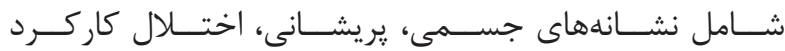

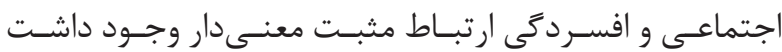

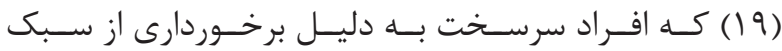

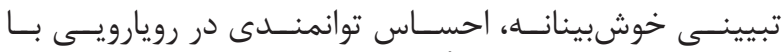

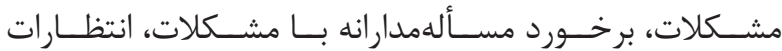

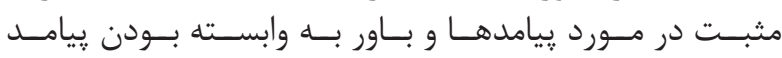

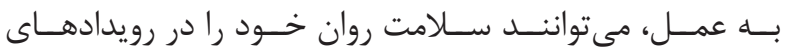

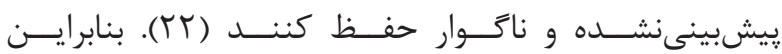

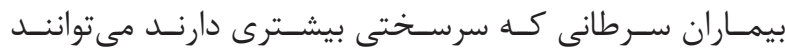

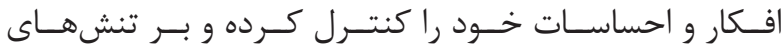

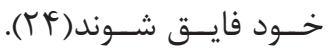

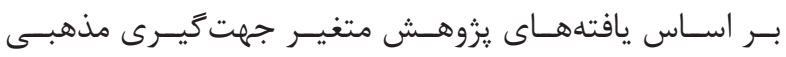

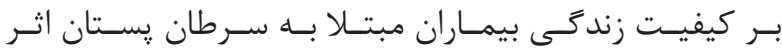

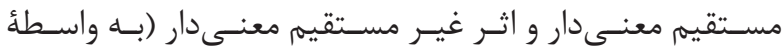

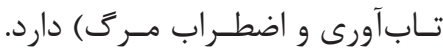

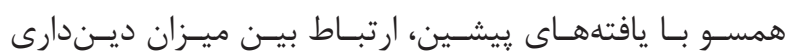

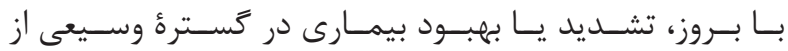

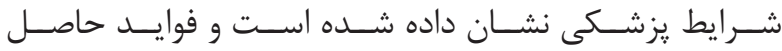

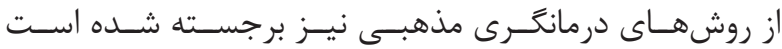

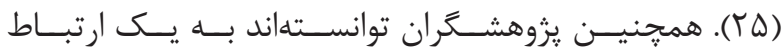

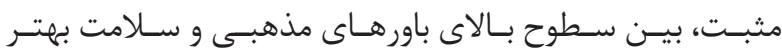

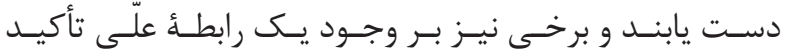




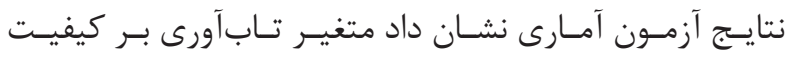

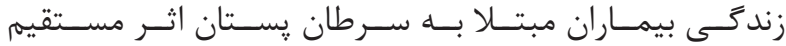

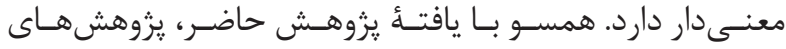

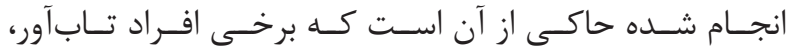

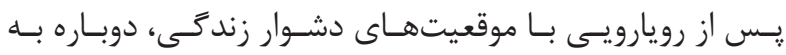

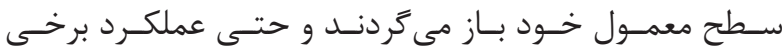

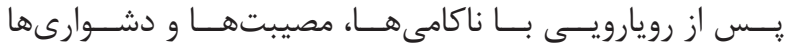

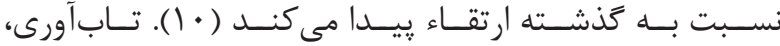

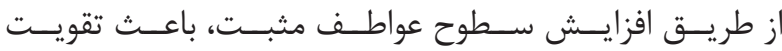

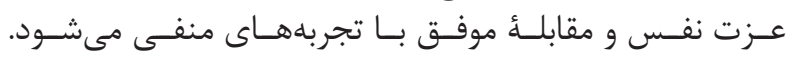

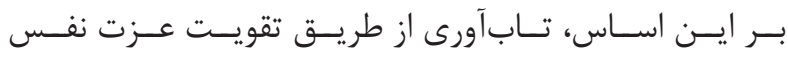

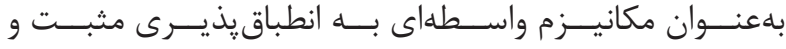

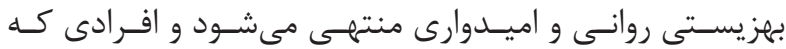

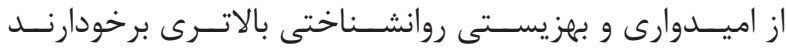

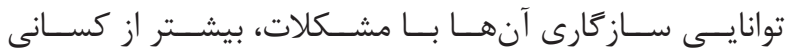

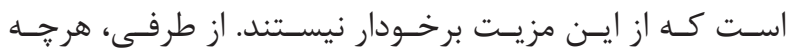

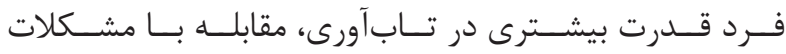

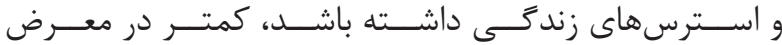

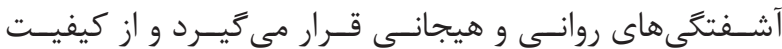

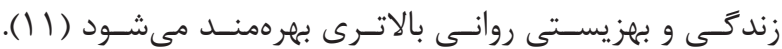

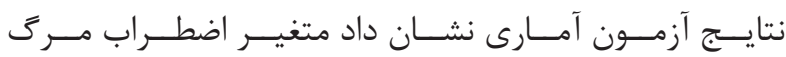

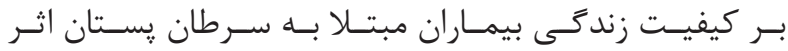
مسـتقيم معنــى دار دارد.

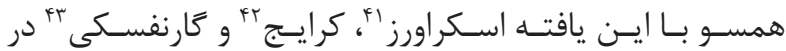

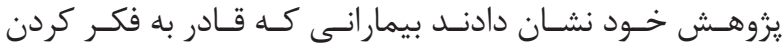

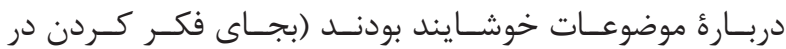

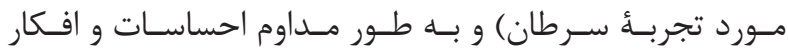

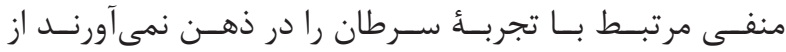

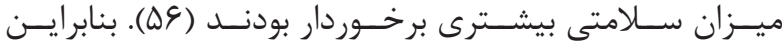

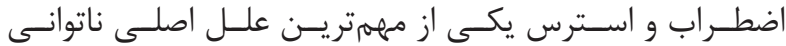

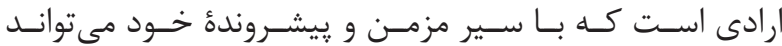

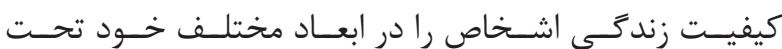

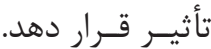

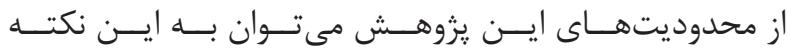

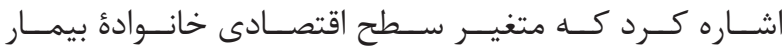

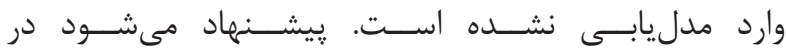

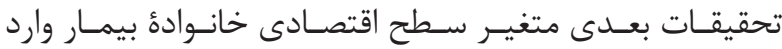

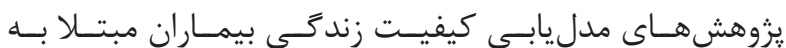

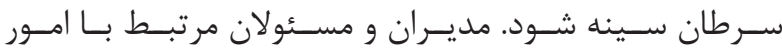

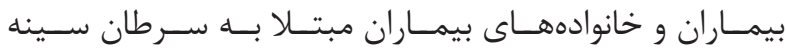

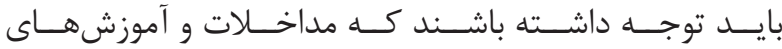

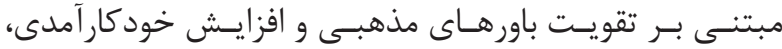

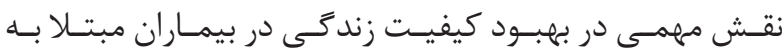

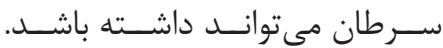

\section{حمايست اجتماعى آنان اسـت.}

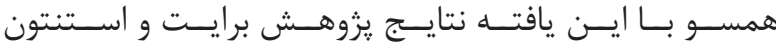

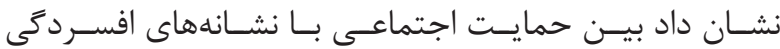

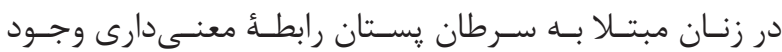

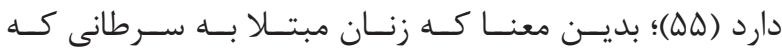

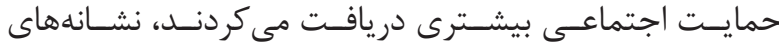

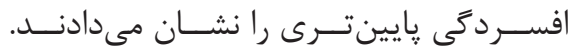

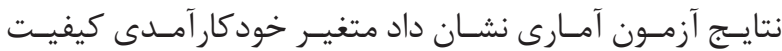

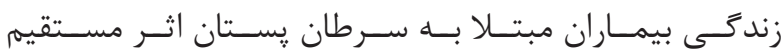

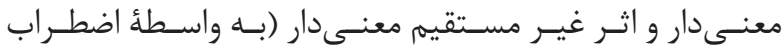

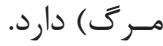

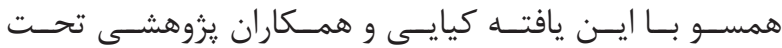

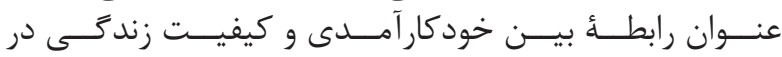

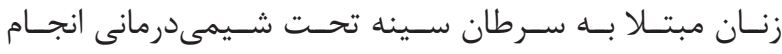

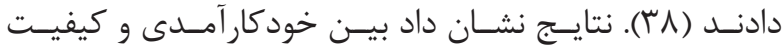

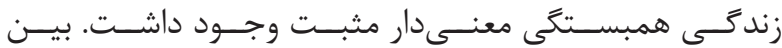

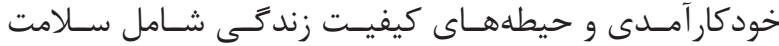

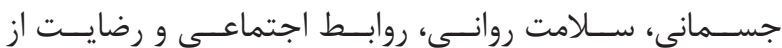

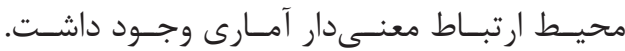

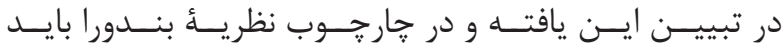

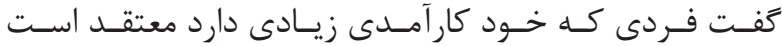

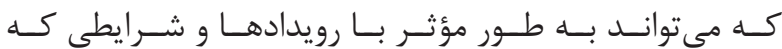

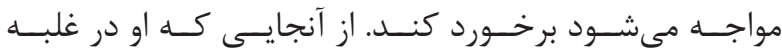

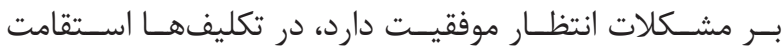

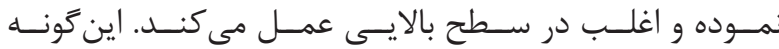

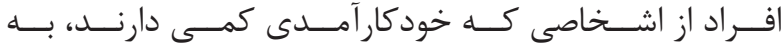

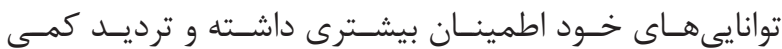

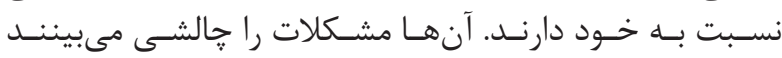

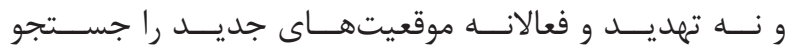

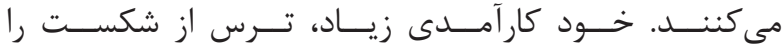

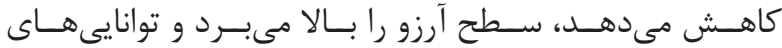

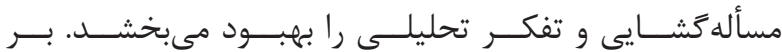

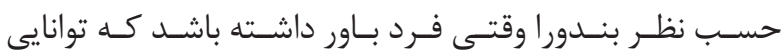

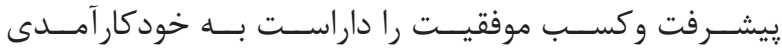

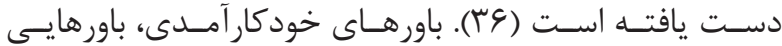

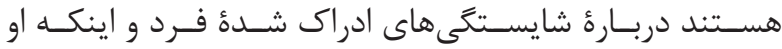

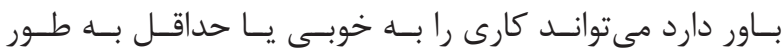

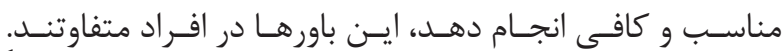

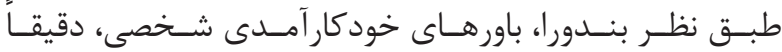

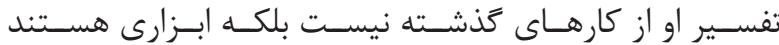

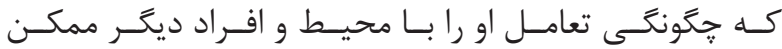

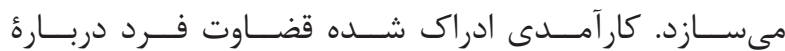

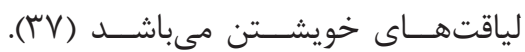


1. Shahbazin S, Mosavi A, Khaledi Pave B, Nasouri M, Azami S. Relationship between psychological status and coping responses in women with breast cancer in Kermanshah in 2012. Clinical Research in Paramedical Sciences. 2012; 2(4): 209-19.

2. Hoseinian S, Yazdi M, Hajian F. Investigating the relationship between goodness and satisfaction of basic psychological needs in women with breast cancer. Imam Hossein University. 2013; 7(20): 90-111.

3. Moradi N, Abdollahzade F, Rahmani A, Zamanzade V, Asodi I, Ghaleban K. The impact of spouses' education on meeting the needs of care support in patients with breast cancer: a clinical trial. Scientific Journal of Hamedan Nursing and Midwifery Faculty. 2013; 21(3): 40-50.

4. Soriano EC, Pasipanodya EC, LoSavio ST, Otto AK, Perndorfer C, Siegel SD, et al. Social constraints and fear of recurrence in couples coping with early stage breast cancer. Health Psychol. 2018; 37(9): 874-84.

5. World Health Organization. Facts on ageing and the life course. Geneva, Switzerland Retrieved. 2014.

6. Trevizan FB, Miyazaki M, Silva Y, Roque C. Quality of life, depression, anxiety and coping strategies after heart transplantation. BJCVS. 2017; 32(3): 162-70.

7. Sanford K, Backer-Fulghum LM, Carson C. Couple resilience inventory: two dimensions of naturally occurring relationship behavior during stressful life events. Psychol Assess. 2016; 28(10): 1243-54.

8. Richardson GE. The metatheory of resilience and resiliency. J Clin Psychol. 2002; 57: 307-21.

9. Campbell-Sills L, Barlow DH, Brown TA, Hofmann SG. Acceptability and suppression of negative emotion in anxiety and mood disorders. Emotion. 2006; 6(4): 587-96.

10. Moskowitz DS. Depression symptoms moderate the association between emotion and communal behavior. J Couns Psychol. 2016; 64(3): 269-79.

11. Liu Y, Wang Z, Li Z. Affective mediators of the influence of neuroticism and resilience on life satisfaction. Personality and individual differences. 2012; 52(7): 833-8.

12. Troy A, Mauss I. Resilience in the face of stress: Emotion regulation as a protective factor. Pathways to Resilience. 2011; 30-44.

13. Yalch MM, Levendosky AA. Influence of betrayal trauma on death anxiety. The Humanistic Psychologist. 2018; 46(4): 390-8.

14. Mansournejad Z, Kajbaf MB. Relationship between religious orientation (external-internal) and gender with death anxiety among students. Journal of Cognitive and Behavioral Sciences. 2012; 2(1): 55-64.

15. Keng SL, Smoski MJ, Robins CJ. Effects of mindfulness on psychological health: a review of empirical studies. Clin Psychol. 2011; 31(6): 1041-56.

16. Folk JB, Loya JM, Alexoudis EA, Tangney JP, Wilson J, Barboza SE. Differences between inmates who attempt suicide and who die by suicide: Staff-identified psychological and treatment-related risk factors. Psychol Serv. 2018; 15(3): 349-56.

17. Blesky J. The psychology of aging. Brooks/Cole Pub. 1999; p. 352.

18. Grady PA, Knebel AR, Draper A. End-of-life issues in AIDS: the research perspective. J R Soc Med. 2001; 94(9): 479-82.

19. Moradi S, Shaker A. The relationship between psychological hardiness and quality of life among teachers of district one high schools of Urmia city. JPBS. 2015; 2: 43-7.

20. Kobasa S, Maddi SR, Puccetti M. Personality and exercise as buffers in the stress-illness relationship. J Behav Med. 1982; 5(4): 391-404.

21. Sandvik AM, Hansen AL, Hystad SW, Johnsen BH, Bartone PT. Psychopathy, anxiety, and resiliencyPsychological hardiness as a mediator of the psychopathy-anxiety relationship in a prison setting. Personality and Individual Differences. 2015; 72: 30-4.

22. Kobasa SC. Stressful life events, personality, and health: An inquiry into hardiness. J Pers Soc Psychol. 1979; 37(1): 1-11.

23. Mobasheri F, Kafi M. The relationship between personality characteristics and psychological hardiness of operative staff in alzahra hospital in Isfahan in 2014. Razi Medical Journal. 2016; 23(143): 47-56.

24. Tarimoradi A. Compares the degree of hardiness and public health between iranian and english women university students. Procedia Soc Behav Sci. 2014; 116: $101-5$.

25. Warren P, Van Eck K, Townley G, Kloos B. Relationships among religious coping, optimism, and outcomes for persons with psychiatric disabilities. 
Psychology of Religion and Spirituality. 2015; 7(2): 91-9.

26. Lahsaei Zadeh A, Azargon Z, Moradi G. Study of religious orientation and mental health of immigrants based on allport \& ross model: case study of sweet palace. Social Sciences Quarterly. 2003; 8: 149.

27. Wen Y-H. Religiosity and death anxiety. The Journal of Human Resource and Adult Learning. 2010; 6(2): $5-7$.

28. Hammer JH, Wade NG, Crugan RT. Valid assessment of spiritual quality of life with the WHOQOL-SRPB BREF across religious, spiritual, and secular persons: A psychometric study. Psychology of Religion and Spirituality. 2019; 4(6): 345-50.

29. Utz S, Breuer J. The relationship between use of social network sites, online social support, and wellbeing: Results from a six-wave longitudinal study. J Media Psychol. 2017; 29(3): 115-25.

30. Hesam M, Asayesh H, Ghorbani M, Shariati A, Nasiri H. Relationship between perceived social support, mental health and life satisfaction in controlled students and self-sacrificing students in governmental universities of Gorgan. Journal of Bioscience and Nursing Faculty of Gorgan. 2011; 1(8): 34-41.

31. Taylor SE, Sherman D, Kim HS, Jarcho J, Takagi K, Dunagan MS. Culture and social support: who seeks it and why? J Pers Soc Psychol. 2007; 87(3): 354-62.

32. Ghodsi AM. Sociological study of the relationship between social support and depression. Tarbiat Modares University. 2003.

33. Dehle C, Landers JE. You cant always get what you want but can you get what you need? Personality traits and social support in marriage. Journal of Social and Clinical Psychology. 2005; 24(7): 1051-76.

34. Rezaei Pour K. The motivation to progress with s tudent self-efficacy. Tehran: Inspirational Light. 2015.

35. Vieno A, Santinello M, Pastore M, Perkins DD. Social support, sense of community in school, and self-efficacy as resources during early adolescence: an integrative model. Am J Community Psychol. 2007; 39: 177-90.

36. Bandura A. Social foundations of though and action: asocial cognitive theory. Englewood Cliffs: Prentic Hall. 1986.

37. Bandura A. Self-efficacy: The exercise of control. New York: Freeman. 1997.
38. Kiyaei MZ, Ferdosi M, Moradi R, Chelongar K, Ahmadzade M, Bahman Ziari N. The relationship between self-efficacy and quality of life in women admitted to breast cancer chemicals. Journal of Qazvin University of Medical Sciences. 2016; 20(2): 58-65.

39. Herts KL, Khaled MM, Stanton AL. Correlates of self-efficacy for disease management in adolescent/ young adult cancer survivors: A systematic review. Health Psychol. 2017; 36(3): 192-205.

40. Bonomi AE, Patrick DL, Bushnell DM, Martin M. Validation of the United States version of the world health organization quality of life (WHOQOL) instrument. J Clin Epidemiol. 2000; 53(1): 19-23.

41. Nejat S, Montazeri A, Holakouie Naieni K, Mohammad K, Majdzadeh S. The world health organization quality of life (whoqol-bref) questionnaire: translation and validation study of the iranian version. SJSPH. 2006; 4(4): 1-12.

42. Connor KM, Davidson JRT. Development of a New Resilience Scale: The connor- davidson resilience scale (CD-RISC). Journal of Depression and Anxiety. 2003; 18: $76-82$.

43. Mohammadi M. Investigating the factors affecting resilience in subjects at risk of substance abuse. University of Rehabilitation Sciences and Social Welfare. 2004.

44. Templer D. Construction of a death anxiety scaleextended. Journal of Death and Dying. 1970; 53(3): 209-26.

45. Rajabi GH, Bahrani M. Factor analysis of death anxiety scale questions. Journal of Psychology. 2002; 5(4): 331-44.

46. Sayini B, Kelaien S. Suicidal behavior in patients diagnosed with cancer of the cervix. The Journal of Crisis Intervention and Suicide Prevention. 1996; 24(4): 168-72.

47. Jomehri F. Relationship between hardiness and tendency to depression and anxiety among female and male students in Tehran University. Allameh Tabatabai University, Tehran. 2001.

48. Allport GW, Ross JM. Personal religious orientation prejudice. Journal of Personality and Social Psychology. 1967; 5: 432-43.

49. Jan Bozorgi M. Religious orientation and mental health. Journal of Faculty of Medicine. 1999; 31(4): 345-50.

50. Zimet G, Dahlem N, Zimet S, Farley G. The 
multidimensional scale of perceived social support. Journal of Personality Assessment. 1988; 52(1): 30-41.

51. Shokri A, Behpajoh A, Daneshvarpour Z, Molaei M, Naghsh Z, Tarkhan R, et al. Factor Structure index of academic expectations. Iranian Psychology Quarterly. 2008; 16: 367-76.

52. Sherer M, Maddux JE, Mercandante B, Prenticedunn S, Jacobs B, Rogers RW. The self-efficacy scale: construction and validation. Psychological Reports. 1982; 51(2): 663-71.

53. Barati S. The investigation between self-efficacy and self-esteem in among high school students. Shahid
Chamran University of Ahvaz. 1998.

54. Fleming R, Baum MM, Gisriel RJ, Gatchel A. Mediating influences of social support on stress at three mile Island. Journal of Human Stress. 1982; 8(3): 14-22.

55. Bright EE, Stanton AL. Prospective investigation of social support, coping, and depressive symptoms: A model of adherence to endocrine therapy among women with breast cancer. Journal of Consulting and Clinical Psychology. 2018; 86(3): 242-53.

56. Schroevers M, Kraaij V, Garnefski N. How do cancer patients manage unattainable personal goals and regulate their emotions? British Journal of Health Psychology. 2010; 13: 351-62. 